\title{
Chorologie, Ecologie et Usages des espèces sauvages apparentées aux légumes feuilles cultivées du genre Solanum L. au Sénégal : Cas de la zone des Niayes
}

\section{Cossi Sosthène Assogbadjo, Master}

Université Cheikh Anta Diop. Département de Biologie végétale. Laboratoire de Botanique et Biodiversité, Dakar-Fann, Sénégal Université d'Abomey-Calavi, Faculté des Sciences Agronomiques, Bénin

\section{Doudou Diop, Docteur}

Université Cheikh Anta Diop. Institut Fondamental d'Afrique Noire/CAD.

Laboratoire de Botanique, Dakar-Fann, Sénégal

\section{Ahuéfa Mauricel Kégbé, Doctorante}

Université Cheikh Anta Diop. Département de Biologie végétale.

Laboratoire de Botanique et Biodiversité, Dakar-Fann, Sénégal

Université d'Abomey-Calavi, Faculté des Sciences Agronomiques, Bénin

Yves Djihounouck, Doctorant

Ndongo Diouf, Docteur

Jules Diouf, Docteur

Ablaye Ngom, Docteur

Mame Samba Mbaye, Professeur

Université Cheikh Anta Diop. Département de Biologie végétale.

Laboratoire de Botanique et Biodiversité, Dakar-Fann, Sénégal

Achille Ephrem Assogbadjo, Professeur

Université d'Abomey-Calavi,

Faculté des Sciences Agronomiques, Cotonou, Bénin

Kandioura Noba, Professeur

Université Cheikh Anta Diop. Département de Biologie végétale. Laboratoire de Botanique et Biodiversité, Dakar-Fann, Sénégal

\section{Doi:10.19044/esj.2021.v17n10p147}

Submitted: 28 October 2020

Accepted: 12 March 2021

Published: 31 March 2021
Copyright 2021 Author(s)

Under Creative Commons BY-NC-ND

4.0 OPEN ACCESS

Cite As:

Assogbadjo C.S., Diop D., Kégbé A.M., Djihounouck Y., Diouf N., Diouf J., Ngom A., Mbaye M.S., Assogbadjo A.E. \& Noba K. (2021). Chorologie, Ecologie et Usages des espèces sauvages apparentées aux légumes feuilles cultivées du genre Solanum L. au Sénégal : Cas de la zone des Niayes. European Scientific Journal, ESJ, 17(10), 147.

https://doi.org/10.19044/esj.2021.v17n10p147 


\section{Résumé}

Le genre Solanum est l'un des plus importants de la flore du Sénégal tant par sa richesse spécifique que par sa valeur socio-économique. Cependant, si les espèces cultivées font l'objet d'étude sous diverses formes, les sauvages sont fréquemment éliminées. L'étude vise à renseigner sur la chorologie, l'écologie et les usages des espèces sauvages apparentées de légumes feuilles cultivées. Des enquêtes ont été menées auprès des populations et les résultats obtenus ont été complétés par la recherche bibliographique et les informations mentionnées dans les parts d'herbier. L'étude révèle que ces espèces occupent des milieux divers. Au Sénégal, elles sont présentes dans toutes les zones agroécologiques avec une grande diversité dans la zone des Niayes. Elles se rencontrent aussi bien en milieux arides où la végétation est de type steppe qu'en milieux humides composées principalement de savanes et affectionnent mieux les sols ferrugineux tropicaux. L'étude ethnobotanique réalisée a révélé cinq types d'utilisation: médicinale $(47,87 \%)$, alimentaire $(27,66 \%)$, culturelle $(14,89 \%)$, fourragère $8,51 \%$ et artisanale $1,06 \%$. Les organes affectés à ces utilisations sont la feuille $(43,36 \%)$ suivie de la racine $(30,97 \%)$, du fruit $(24,78 \%)$ et de la tige $(0,88 \%)$. La connaissance des espèces favorise une meilleure valorisation de celles qui vivent dans des écosystèmes en proie à une forte urbanisation.

Mots-clés : Solanum, chorologie, écologie, Sénégal 


\title{
Chorology, Ecology and Uses of wild species related to cultivated leaf vegetables of the genus Solanum L. in Senegal: Case of the Niayes zone
}

\section{Cossi Sosthène Assogbadjo, Master}

Université Cheikh Anta Diop. Département de Biologie végétale. Laboratoire de Botanique et Biodiversité, Dakar-Fann, Sénégal Université d'Abomey-Calavi, Faculté des Sciences Agronomiques, Bénin

\section{Doudou Diop, Docteur}

Université Cheikh Anta Diop. Institut Fondamental d'Afrique Noire/CAD. Laboratoire de Botanique, Dakar-Fann, Sénégal

\section{Ahuéfa Mauricel Kégbé, Doctorante}

Université Cheikh Anta Diop. Département de Biologie végétale.

Laboratoire de Botanique et Biodiversité, Dakar-Fann, Sénégal Université d'Abomey-Calavi, Faculté des Sciences Agronomiques, Bénin

\section{Yves Djihounouck, Doctorant}

Ndongo Diouf, Docteur

Jules Diouf, Docteur

Ablaye Ngom, Docteur

Mame Samba Mbaye, Professeur

Université Cheikh Anta Diop. Département de Biologie végétale.

Laboratoire de Botanique et Biodiversité, Dakar-Fann, Sénégal

Achille Ephrem Assogbadjo, Professeur

Université d'Abomey-Calavi,

Faculté des Sciences Agronomiques, Cotonou, Bénin

\section{Kandioura Noba, Professeur}

Université Cheikh Anta Diop. Département de Biologie végétale. Laboratoire de Botanique et Biodiversité, Dakar-Fann, Sénégal

\begin{abstract}
The genus Solanum is one of the most important of the flora of Senegal both for its specific richness and its socio-economic value. However, while cultivated species are studied in various forms, wild species are frequently eliminated. The study aims to provide information on the chorology, ecology and uses of wild relatives of cultivated leafy vegetables. Population surveys were conducted and the results obtained were supplemented by literature search and information mentioned in the herbarium parts. The study revealed that these species occupy diverse environments. In Senegal, they are present in all agro-ecological zones with a great diversity in the Niayes zone. They are found in arid environments where the vegetation is
\end{abstract}


steppe type as well as in humid environments composed mainly of savannah and are more fond of tropical ferruginous soils. The ethnobotanical study carried out revealed five types of use: medicinal (47.87\%), food (27.66\%), cultural $(14.89 \%)$, fodder $(8.51 \%)$ and artisanal $(1.06 \%)$. The organs assigned to these uses are the leaf $(43.36 \%)$ followed by the root $(30.97 \%)$, the fruit $(24.78 \%)$ and the stem $(0.88 \%)$. The knowledge of the species favors a better valorization of those living in ecosystems in prey to a strong urbanization.

Keywords: Solanum, chorology, ecology, Senegal

\section{Introduction}

Les espèces sauvages apparentées aux plantes cultivées constituent un énorme réservoir de variabilité génétique qui est utilisable dans les programmes de sélection végétale et indispensable pour améliorer la sécurité alimentaire, stimuler la production agricole et maintenir la productivité (Hunter \& Heywood, 2011). Parmi les nombreuses espèces sauvages apparentées aux plantes cultivées utiliser par l'homme se trouvent les espèces du genre Solanum. Ce genre est le plus diversifié de la famille des Solanaceae et l'un des dix plus grands genres des espèces végétales (Frodin, 2004). Il est en réalité difficile d'établir sa taille exacte, mais le nombre d'espèces qu'il renferme est estimé à plus de 1350 (Samuels, 2015). Les espèces sauvages apparentées sont présentes dans les écosystèmes naturels et semi-naturels Vollbrecht \& Sigmon (2005) et interviennent pour répondre aux besoins d'une population mondiale de plus en plus croissante représentant un des défis majeurs de notre époque. Elles jouent un rôle important dans les régimes alimentaires des populations (Akpavi et al., 2011) et occupent une place importante dans le maintien de l'équilibre alimentaire pour celles défavorisées (Clay, 2002). En plus, les espèces du genre Solanum sont utilisées à des fins médicinales (Alsherbiny et al., 2015). En effet, leur consommation comme alicament pourrait aider à prévenir et à traiter beaucoup de maladies (Jain et al., 2011). Cependant, en dépit de leur importance, plusieurs menaces telles que les changements climatiques, la désertification, les pressions démographiques pèsent sur ces ressources (Ozer et al., 2010 ; Maxted et Kell, 2009). En effet, la zone des Niayes, milieu atypique bordant la frange littorale du Sénégal caractérisée par une végétation jadis luxuriante est aujourd'hui sujette à une dégradation sans précédent et fortement menacée de disparition sous l'effet d'une urbanisation accélérée (Diallo et al., 2009). La connaissance de ces espèces reste un impératif pour des stratégies de conservation et de valorisation. A part les études botaniques de Berhaut $(1967$; 1988) et Kerharo et al. (1974) sur la caractérisation de la flore globale et sur la pharmacopée Sénégalaise traditionnelle, les espèces sauvages apparentées du genre Solanum ont fait l'objet de très peu d'études scientifiques. C'est dans ce cadre 
que nous envisageons de mener cette étude pour apporter des données récentes sur la diversité des espèces mais aussi leur importance pour la population locale. En d'autres termes, elle se concentre sur la répartition géographique, l'écologie et les usages faits des espèces sauvages apparentées aux légumes feuilles cultivés du genre Solanum par les populations. Plus spécifiquement, il s'agira :

- d'établir la distribution des espèces sauvages apparentées aux légumes feuilles cultivés du genre Solanum,

- d'établir les corrélations entre la distribution géographique et les facteurs écologiques ;

- d'identifier les usages de ces espèces par les populations locales.

\section{Méthodologie}

\section{Répartition géographique et écologie des espèces au Sénégal.}

Le Sénégal est situé à l'extrême Ouest du continent Africain, entre $12^{\circ} 5$ et $16^{\circ} 5$ de latitude Nord puis $11^{\circ} 5$ et $17^{\circ} 5$ de longitude Ouest. Il couvre une superficie de $196.712 \mathrm{~km}^{2}$ et est limité au Nord par la Mauritanie, à l'Est par le Mali, au Sud par la Guinée Conakrie et la Guinée Bissau et à l'Ouest par l'Océan Atlantique sur une façade de $700 \mathrm{~km}$. La République de Gambie constitue une enclave de plus de $300 \mathrm{~km}$ à l'intérieur du territoire sénégalais (ANACIM, 2018). En fonction de sa latitude tropicale, le Sénégal appartient à la zone de transition entre la ceinture périphérique des déserts tropicaux (climat sahélien) et la zone équatoriale centre africaine (climat guinéen) ( Ndao, 2012). Ce positionnement permet au pays de disposer d'une diversité d'écosystèmes riches en espèces (MEPN, 1997).

Selon Maxted et al. (2006), on entend par «parent sauvage », toute espèce appartenant au même genre que l'espèce cultivée mais qui pousse de manière naturelle. Se basant sur cette définition, 15 espèces sauvage apparentées aux légumes feuilles cultivés du genre Solanum ont été recensées au Sénégal. La recherche documentaire réalisé porte sur la répartition géographique et les conditions écologiques des espèces (Hutchinson et al., 1958 ; Berhaut, 1979 ; Adam, 1981 ; Berhaut, 1988 ; Kerharo et al., 1974 ; Bukenya \& Carasco, 1995 ; Gbile \& Adesina, 1988 ; Lebrun et al., 1992 ; Alsherbiny et al., 2015; Samuels et al., 2015). Des sites internet ont été consultés (base de données des plantes d'Afrique: http://www.villege.ch/musinfo/bd/cjb/africa/, https://www.prota4u.org, la base de données Global Biodiversity Information Facility: https://www.gbif.org/fr/) et les données obtenus ont été complétées par les indications fournies sur les échantillons des herbiers de l'Institut Fondamental d'Afrique Noire (IFAN) et du Département de Biologie Végétale de l'Université Cheikh Anta Diop (UCAD) à Dakar. Les aires de répartitions des espèces végétales en Afrique tropicale, représentées par les subdivisions phytogéographiques proposées par 
Lebrun (1947) et cité dans Diop et al. (2012) ont été utilisées pour caractériser la répartition des espèces du genre Solanum au niveau continental. La superposition de la carte de l'Afrique avec ces subdivisions phytogéographiques, sur celle contenant les limites des États d'Afrique, a permis d'apprécier l'aire de distribution géographique de chacune des espèces étudiées. Dans cette étude, trois facteurs écologiques ont été retenus. Il s'agit du climat, de la végétation, et du sol qui représentent des facteurs importants influençant la répartition des espèces végétales. La carte des zones climatiques utilisée est celle défini par Mbow (2009) alors que les caractéristiques des types de végétation et de sol ont été fournies par l'observatoire du Centre de Suivi Ecologique (2007).

Une approche de géoréférencement basée sur la sélection de cartes avec le logiciel ARCGIS a été utilisée. Celle-ci consiste à une projection des coordonnées géographiques sur une carte géoréférencée permettant de délimiter les aires de répartition des espèces.

\section{Ethnobotanique}

Une visite de prospection a été menée en collaboration avec la population locale afin de choisir les régions à visiter tout en restant dans une logique de présence des espèces du genre Solanum dans les lieux. Ainsi, trois régions (Dakar, Thiès et Saint Louis au Sénégal) des quatre constituants les Niayes ont été retenues pour les enquêtes ethnobotaniques. (Tableau 1)

Tableau 1 : Communes et villages retenues dans chaque région pour l'étude

\begin{tabular}{lll}
\hline Régions & Communes & Villages \\
\hline Dakar & Sangalkam et Mbao & Sangalkam, Noflaye, Mbao et Keur Massa \\
\hline Thiès & Méouane et Pambal & $\begin{array}{l}\text { Daho Diop, Dougname, Darou khoudoss et Baïty- } \\
\text { bacar }\end{array}$ \\
\hline $\begin{array}{l}\text { Saint- } \\
\text { Louis }\end{array}$ & $\begin{array}{l}\text { Gandon et } \text { Ross } \\
\text { Béthio }\end{array}$ & Rao, Kallassane, Ndiawdoune et Darou-salam \\
\hline
\end{tabular}

Pour mener à bien nos activités, un questionnaire teste a été administré sur des personnes choisies de manière aléatoire (Alves \& Alves, 2011) afin de s'assurer de la bonne compréhension des questions par chacun des acteurs (interprète, répondant et enquêteur). Ce processus nous a permis de limiter la déformation des informations lors de la traduction orale et de la transcription. Dans chaque région, il était question de visiter quatre villages autrement dit, deux villages par commune en fonction de leurs fort potentiel en production agricole notamment maraîchères.

La méthode non probabiliste par convenance a été utilisée comme méthode d'échantillonnage. À cet effet, le choix des personnes a été basé sur des critères précis. Il s'agissait de rencontrer le chef du village qui est la personne morale de sa communauté. Après explication de l'objectif de notre 
visite, il nous établissait une liste de personnes à interroger. Suivant l'âge, le sexe et l'activité socio- professionnelle, 10 personnes par village ont été retenues pour l'enquête étant donné que la différence du nombre de personnes engagées dans ces domaines d'activités entre les différents villages n'est pas très importante (variant entre 8 et 12). Ces entrevues ont été appliquées majoritairement auprès de personnes majeures autochtones et de préférence des hommes pour leurs connaissances avec les espèces sauvages. Pour éviter toute confusion sur la reconnaissance des espèces par les populations, des photos de chaque espèce ont été montrées à ces derniers.

Les données ont été collectées à travers des entretiens semi-structurés à l'aide d'une fiche d'enquête comprenant deux parties : une partie relative aux informations socio-démographiques de l'informateur (nom et prénoms, âge, situation matrimoniale, ethnie, catégorie socioprofessionnelle...), une autre partie relative aux informations sur les espèces sauvages du genre Solanum. Toutes les personnes ont été interrogées individuellement. L'identification des espèces récoltées a été faite soit sur place, soit au laboratoire de botanique de l'IFAN / UCAD et au Laboratoire de Botanique et Biodiversité du département de Biologie Végétale à l'aide des flores illustrées de Berhaut $(1967 ; 1979 ; 1988)$ et des collections d'herbiers Dakar et de IFAN. La nomenclature adoptée est celle de la base de données du Conservatoire et Jardin Botanique (C.J.B) de la ville de Genève (Lebrun et Stork, $1997 ; 1995 ; 1992 ; 1991)$. Le nom scientifique de chaque espèce a été transcrit en langue locale à l'aide des documents spécialisés (Adam, 1970 ; Berhaut, 1967).

Le dépouillement des données a été effectué avec le logiciel d'analyse statistique Epi Info. Les données collectées ont été traitées et représentées graphiquement grâce au tableur Excel. La fréquence de citation $(\mathrm{Fc})$ de chaque plante a été déterminée suivant la formule indiquée par (Fah et al 2013).

$$
F C=\frac{\mathrm{n}}{\mathrm{N}}
$$

Avec $: \mathrm{n}=$ Nombre de citations de l'espèce et $\mathrm{N}=$ Nombre total de citations de toutes les espèces $(\mathrm{N})$.

Une Analyse Factorielle des Correspondances (AFC) a été réalisée avec le logiciel R (R Core Team, 2017) avec le package factoMiner (Version $: 1.0 .5)$ pour établir la relation entre les espèces et les régions prospectées.

\section{Résultats \\ Répartition géographique et écologie Répartition géographique}

Le Tableau 2 récapitule la répartition géographique des espèces sauvages apparentées de légumes feuilles cultivés du genre Solanum dans le monde, en Afrique et au Sénégal. 
A l'échelle mondiale, les résultats montrent que :

- une seule espèce est cosmopolite. Il s'agit de $S$. nigrum présente sur tous les continents ;

- douze espèces, $S$. forskalii, $S$. terminale, $S$. incanum, $S$. aculeatissimum, S. americanum, $S$. torvum, S. anguivi, S. erianthum, S. dasyphyllum, S. scabrum S. sisymbriifolium et $S$. seafortianum sont signalées dans trois continents (Afrique, Amérique et Asie) ;

- $\quad$ sept espèces : S. erianthum, S. anguivi, S. torvum, S. aculeatissimum, $S$. incanum, S. americanum et $S$. scabrum ont été signalée en Europe.

- deux espèces : S. anomalum et $S$. cerasiferum sont signalées dans deux continents (Afrique, Amérique).

En Afrique, les espèces étudiées sont Plurirégionales Africaine.

Tableau 2 : Répartition géographique des espèces du genre Solanum rencontrées au Sénégal

\begin{tabular}{|c|c|c|c|}
\hline Espèces & Monde & Afrique & Sénégal \\
\hline S. aculeatissimum & $\begin{array}{l}\text { Afrique }{ }^{2,3,4,5} \\
\text { Asie }{ }^{4,5} \\
\text { Amérique }^{4,5} \\
\text { Europe }^{4}\end{array}$ & $\begin{array}{l}\text { Plurirégionales Africaines }{ }^{3,4,5} \\
\text { (Afrique de l'Est et de l'Ouest) }\end{array}$ & $\begin{array}{l}\text { Niayes }{ }^{2,3}, \text { Fleuve } \\
\text { Sénégal } \\
\text { 2,3 }, \text { Bassin } \\
\text { arachidier }^{3} \text {, Sénégal } \\
\text { oriental }^{3}\end{array}$ \\
\hline S. americanum & $\begin{array}{l}\text { Afrique }^{2,3,4,5} \\
\text { Amérique } \\
\text { Asie } \\
4,5 \\
\text { Europe }^{4}\end{array}$ & Plurirégionales Africaines ${ }^{1,4}$ & Niayes 1,3 \\
\hline S. nigrum & Cosmopolite $1,2,3,4,5$ & $\begin{array}{l}\text { Plurirégionales Africaines }{ }^{1,4,5} \\
\text { (Afrique de l'Est, de l'Ouest du } \\
\text { nord ...) }\end{array}$ & $\begin{array}{l}\text { Niayes }{ }^{1,2,3}, \text { Fleuve } \\
\text { Sénégal }^{2,3}, \text { Sénégal } \\
\text { oriental }^{3}, \text { Ferlo }^{2,3}\end{array}$ \\
\hline S. torvum & $\begin{array}{l}\text { Afrique }{ }^{2,5} \\
\text { Amérique }{ }^{4,5} \\
\text { Asie }{ }^{4,5} \\
\text { Europe }^{4}\end{array}$ & $\begin{array}{l}\text { Plurirégionales Africaines }{ }^{3,4} \\
\text { (Afrique de l'Ouest ; Afrique } \\
\text { centrale) }\end{array}$ & Niayes $^{3}$ \\
\hline S. incanum & $\begin{array}{l}\text { Afrique }{ }^{1,2,3,5} \\
\text { Amérique }{ }^{4,5} \\
\text { Asie }{ }^{4,5} \\
\text { Europe }^{4}\end{array}$ & $\begin{array}{l}\text { Plurirégionales Africaines }{ }^{3,4} \\
\text { (Afrique de l'Est et de l'Ouest) }\end{array}$ & $\begin{array}{l}\text { Niayes }{ }^{2,3}, \text { Fleuve } \\
\text { Sénégal } \\
\text { 2,3 } \\
\text { arachidier }{ }^{3} \text {, Sénégal } \\
\text { oriental }^{3,4} \text { Casamance }^{3}\end{array}$ \\
\hline S. anguivi & $\begin{array}{l}\text { Afrique }^{2,4,5} \\
\text { Amérique } \\
\text { Asie } \\
\text { Europe }^{4}\end{array}$ & Plurirégionales Africaines ${ }^{4,5}$ & Bassin arachidier ${ }^{3}$ \\
\hline S. terminale & $\begin{array}{l}\text { Afrique }{ }^{2,3,4} \\
\text { Amérique } \\
\text { Asie }^{4,5}\end{array}$ & $\begin{array}{l}\text { Plurirégionales Africaines } 4,5 \\
\text { (Se rencontre depuis la Guinée } \\
\text { jusqu'en Ethiopie et vers le sud } \\
\text { jusqu'en Angola et en Afrique } \\
\text { du Sud) }\end{array}$ & Niayes $^{3}$ \\
\hline S. anomalum & $\begin{array}{l}\text { Afrique }{ }^{2,3,5} \\
\text { Amérique }^{4,5}\end{array}$ & $\begin{array}{l}\text { Plurirégionales Africaines }{ }^{4,5} \\
\text { (Sénégal, Bénin Sierra Leone } \\
\text { jusqu'au sud du Nigeria, au } \\
\text { Cameroun et en R.D. du Congo) }\end{array}$ & Niayes ${ }^{3}$ \\
\hline
\end{tabular}




\begin{tabular}{|c|c|c|c|}
\hline S. erianthum & $\begin{array}{l}\text { Afrique }^{2,5} \\
\text { Amérique }^{2,3,5} \\
\text { Asie }^{4,5} \\
\text { Europe }^{4}\end{array}$ & $\begin{array}{l}\text { Plurirégionales Africaines }{ }^{4,5} \\
\text { (Sénégal, Bénin, Cote d'ivoire, } \\
\text { Uganda, Nigeria) }\end{array}$ & Niayes $^{3}$ \\
\hline S. cerasiferum & $\begin{array}{l}\text { Afrique }^{2,3,5} \\
\text { Amérique }\end{array}$ & $\begin{array}{l}\text { Plurirégionales Africaines }{ }^{4,5} \\
\text { (Sénégal, Cote d'ivoire, } \\
\text { Uganda, Kenya) }\end{array}$ & $\begin{array}{l}\text { Niayes }{ }^{2,3}, \text { Bassin } \\
\text { arachidier }^{3} \text {, Sénégal } \\
\text { oriental }^{1,3}, \text { Ferlo }^{3}\end{array}$ \\
\hline S. dasyphyllum & $\begin{array}{l}\text { Afrique } 2,3,5 \\
\text { Amérique } \\
\text { Asie }^{4}\end{array}$ & $\begin{array}{l}\text { Plurirégionales Africaines }{ }^{4,5} \\
\text { (Sénégal, Bénin, Cote d'ivoire, } \\
\text { Uganda) }\end{array}$ & $\begin{array}{l}\text { Bassin arachidier }{ }^{3} \\
\text { Ferlo }^{3}, \text { Casamance }^{3}\end{array}$ \\
\hline S. scabrum & $\begin{array}{l}\text { Afrique }^{2,4,5} \\
\text { Asie }^{4,5} \\
\text { Amérique }^{4,5} \\
\text { Europe }^{4}\end{array}$ & $\begin{array}{l}\text { Plurirégionales Africaines } \\
\text { (Afrique de l'Est et de l'Ouest) }\end{array}$ & Niayes $^{3}$ \\
\hline S. sisymbriifolium & $\begin{array}{l}\text { Afrique }{ }^{2,4,5} \\
\text { Amérique } \\
\text { Asie }^{5}\end{array}$ & $\begin{array}{l}\text { Plurirégionales Africaines }{ }^{3,4,5} \\
\text { (Sénégal, Bénin, Cote d'ivoire, } \\
\text { Uganda, Mali) }\end{array}$ & Sénégal oriental ${ }^{1,3}$ \\
\hline S. seafortianum & $\begin{array}{l}\text { Afrique }^{2,4} \\
\text { Asie }^{4} \\
\text { Amérique }^{4,5}\end{array}$ & $\begin{array}{l}\text { Plurirégionales Africaines }{ }^{3,4} \\
\text { (Afrique de l'Est et de l'Ouest) }\end{array}$ & Fleuve Sénégal ${ }^{3}$ \\
\hline S. forskalii & $\begin{array}{l}\text { Afrique } 2,3,4 \\
\text { Amérique } \\
\text { Asie }^{4}\end{array}$ & $\begin{array}{l}\text { Plurirégionales Africaines }{ }^{4} \\
\text { (Sénégal, Cote d'ivoire, } \\
\text { Uganda, Malawi) }\end{array}$ & $\begin{array}{l}\text { Niayes }{ }^{3}, \text { Fleuve } \\
\text { Sénégal }^{3}\end{array}$ \\
\hline
\end{tabular}

1 : observations personnelles, 2 : Berhaut, 1979 ; Berhaut, 1988 ; 3 : fiches d'Herbier ; 4 : http://www.gbif.org, (02.01.2020), 5 : https://www.prota4u.org (02.01.2020)

Les Figures 1 et 2 présentent respectivement, la répartition géographique et les zones agro-écologiques couvertes par chacune des espèces sauvages apparentées de légumes feuilles cultivés du genre Solanum au Sénégal.

En ce qui concerne la diversité spécifique, elle est plus marquée dans la zone des Niayes où 11 espèces sont signalées, suivie du Sénégal oriental avec 6 espèces, du Fleuve Sénégal et du Bassin arachidier avec 5 espèces chacune, du Ferlo avec 3 espèces et enfin de la Casamance avec 2 espèces. Pour ce qui est de la répartition agro-écologique :

- S. incanum est l'espèce la plus répandue car présente dans toutes les zones agro-écologiques du Sénégal.

- S. nigrum, S. dasyphyllum, S. aculeatissimum et S. cerasiferum sont les espèces moyennement répandues car retrouvées dans plus de deux zones agro-écologiques.

- S. americanum, S. torvum, S. anguivi, S. terminale, S. anomalum, S. erianthum, $S$. scabrum, $S$. sisymbriifolium, $S$. seafortianum et $S$. forskalii constituent les espèces à répartition faible car confinées dans une ou deux zones agro-écologiques. 


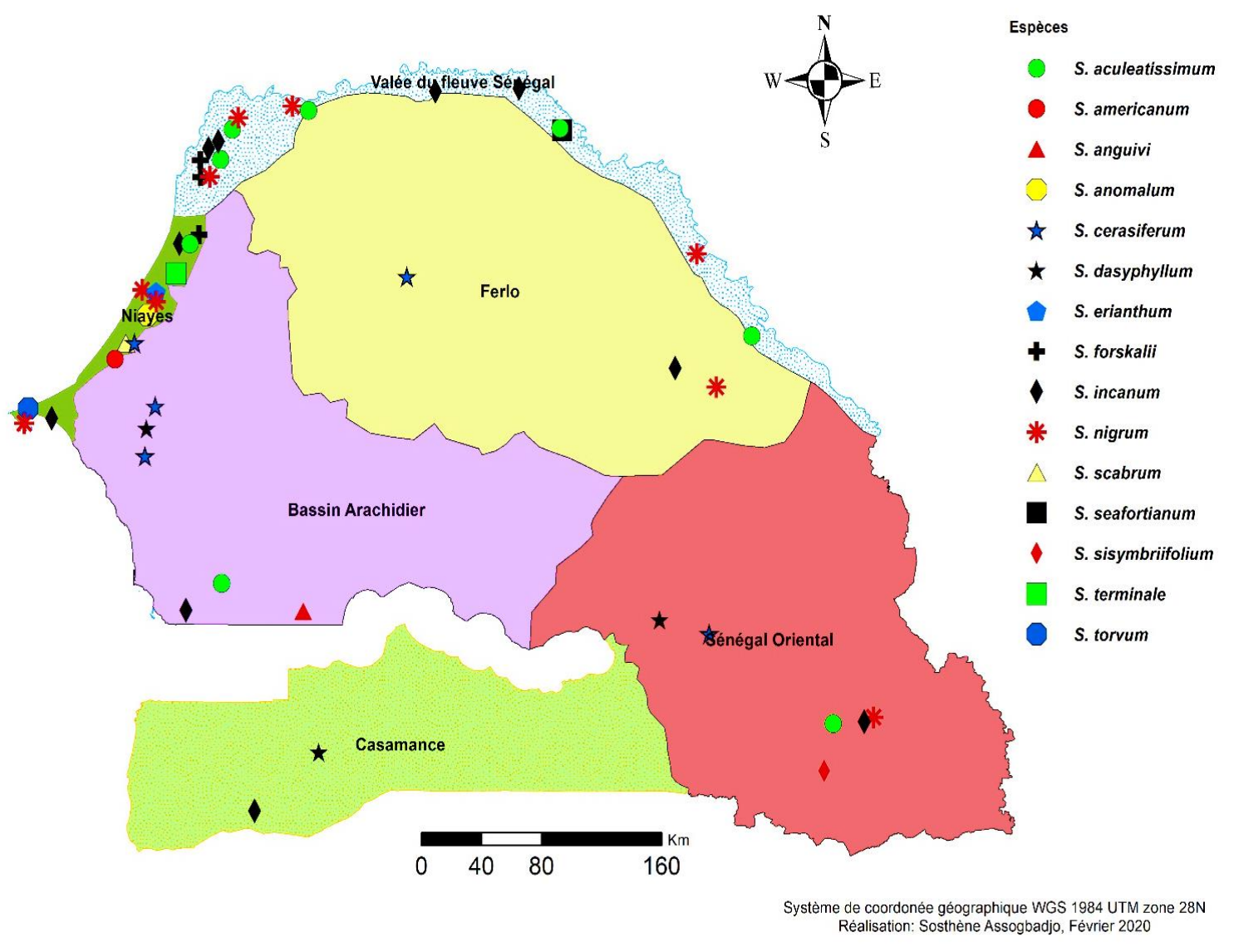

Figure 1 : Répartition géographiques des espèces en fonctions des zones agro-écologiques 

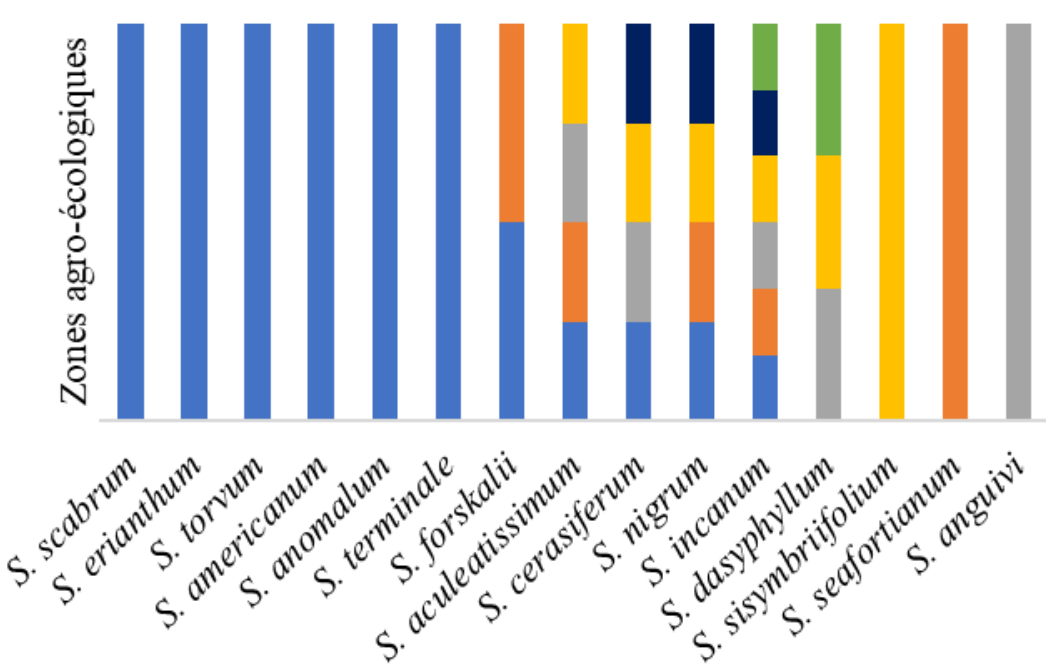

Espèces
- Casamance

- Ferlo

- Sénégal Oriental

- Bassin Arachidier

- Fleuve Sénégal

- Niaye

Figure 2 : Zones agro-écologiques couvertes par chacune des espèces au Sénégal

\section{Ecologie des espèces}

Au niveau des zones phytogéographiques, si dans leur intégralité les espèces sauvages apparentées de légumes feuilles cultivées du genre Solanum supportent un large éventail de conditions environnementales. Il convient néanmoins de mentionner que certaines d'entre-elles ont des caractéristiques spécifiques qui les lient à une zone phytogéographique. Ainsi, deux groupes peuvent être définis en fonction de leurs aires de répartition (Figure 3) :

- des espèces à large spectre de distribution; il s'agit de S. nigrum, S. incanum, S. cerasiferum, S. dasyphyllum et $S$. aculeatissimum qui sont rencontrées au nord et au sud du pays dans différentes zones phytogéographiques telles que les zones sahélienne, sahélo-soudanienne et guinéenne ; 
- des espèces à spectre de distribution moyen à faible ; il s'agit de S. scabrum, S. erianthum, S. torvum, S. terminale, $S$. americanum et $S$. anomalum qui sont d'affinité sahélo-soudanienne, de $S$. seafortianum rencontrée dans la zone sahélienne, de $S$. anguivi et $S$. sisymbriifolium circonscrite dans la zone soudanienne et de S. forskalii retrouvée dans les zones sahélienne et sahélo-soudanienne.

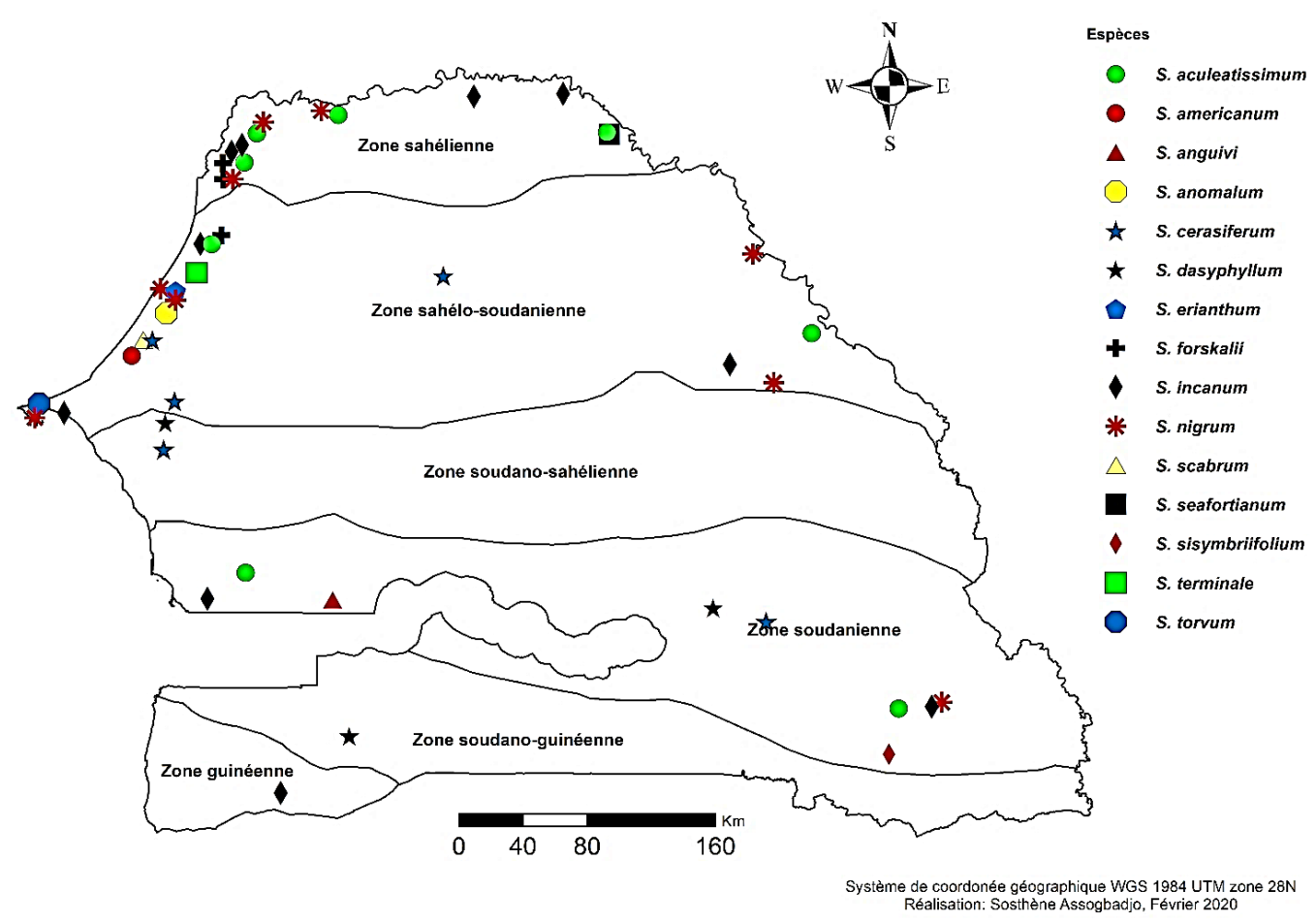

Figure 3 : Répartition des espèces en fonction des zones phytogéographiques du Sénégal 
A l'image des zones phytogéographiques, les espèces sauvages apparentées de légumes feuilles cultivées du genre Solanum sont présentes dans presque tous les types de végétation du Sénégal constitués majoritairement de savanes et de steppes (Ngom et al., 2016). En effet, certaines espèces se rencontrent à la fois en milieux arides où la végétation est de type steppe (au nord) et humides (au sud) composées principalement de forêts et de savanes boisées (Tableau 3). C'est le cas de: S. nigrum, $S$. incanum, $S$. cerasiferum, $S$. dasyphyllum et $S$. aculeatissimum. Pour d'autres espèces, la particularité réside dans leur confinement au Nord ou au Sud du pays. Au nord, il s'agit de S. scabrum, S. erianthum, S. torvum, S. terminale, $S$. americanum et $S$. seafortianum retrouvées dans des savanes arbustives et au niveau des jachères. Au Sud, c'est le cas de $S$. anguivi rencontrée dans les savanes boisées et de $S$. sisymbriifolium retrouvée dans les forêts humides. (Tableau 3)

Du point de vue pédologie, les résultats de la présente étude montrent que les espèces étudiées, prises dans leur intégralité, poussent sur la plupart des sols du pays (Tableau 3). En effet, on les trouve sur des :

- (i) sols ferrugineux tropicaux : S. aculeatissimum, S. americanum, $S$. nigrum, S. torvum, $S$. anguivi, $S$. anomalum, $S$. erianthum, $S$. cerasiferum, $S$. dasyphyllum, S. scabrum et $S$. forskalii ;

- (ii) sols brun subarides: S. nigrum, S. incanum, S. anomalum; $S$. cerasiferum, S. dasyphyllum, et S. seafortianum;

- (iii) sols halomorphes : S. aculeatissimum, S. nigrum, S. incanum et $S$. sisymbriifolium ;

- (iv) sols hydromorphes: $S$. americanum, $S$. anomalum et $S$. dasyphyllum;

- (v) sols rouge brun : S. terminale et S. forsskalii ;

- (vi) sols ferralitiques : S. incanum.

La Figure 4 présente le nombre d'espèces signalées par type de sol au Sénégal. On observe que les espèces étudiées affectionnent mieux les sols ferrugineux tropicaux (11 espèces) qui sont prédominantes au Sénégal et conviennent à une gamme plus large de cultures du fait de leur plus grande richesse minérale.

Moins fréquent, elles poussent sur les sols rouges bruns et les sols ferralitiques qui sont à fertilité relativement faible. 
Tableau 3 : Caractéristiques écologiques des espèces sauvages apparentées de légumes feuilles cultivés du genre Solanum au Sénégal.

\begin{tabular}{|c|c|c|c|}
\hline \multirow[t]{2}{*}{ Espèces } & \multicolumn{3}{|c|}{ Caractères écologiques } \\
\hline & Biotope & Climat & Pédologie \\
\hline S. aculeatissimum & $\begin{array}{l}\text { Galerie forestière, } \\
\text { Savanes arbustives }\end{array}$ & Climat soudanien & $\begin{array}{l}\text { Sols : Halomorphes et } \\
\text { Ferrugineux tropicaux }\end{array}$ \\
\hline S. americanum & $\begin{array}{l}\text { Savanes arbustives, } \\
\text { Jachères }\end{array}$ & $\begin{array}{l}\text { Climat sahélo- } \\
\text { soudanien }\end{array}$ & $\begin{array}{l}\text { Sols : Hydromorphes } \\
\text { Ferrugineux tropicaux }\end{array}$ \\
\hline S. nigrum & $\begin{array}{l}\text { Galeries forestières, } \\
\text { Savanes arbustives, } \\
\text { Steppes }\end{array}$ & Climat soudanien & $\begin{array}{l}\text { Sols : Brun subaride, } \\
\text { Ferrugineux } \\
\text { tropicaux } \\
\text { Halomorphe }\end{array}$ \\
\hline S. torvum & $\begin{array}{l}\text { Savanes arbustives, } \\
\text { Jachères }\end{array}$ & $\begin{array}{l}\text { Climat sahélo- } \\
\text { soudanien }\end{array}$ & $\begin{array}{l}\text { Sols ferrugineux } \\
\text { tropicaux }\end{array}$ \\
\hline S. incanum & $\begin{array}{l}\text { Forêt humide, } \\
\text { Savane boisées, } \\
\text { Steppes }\end{array}$ & Climat soudanien & $\begin{array}{l}\text { Sols : Bruns } \\
\text { subarides, } \\
\text { Ferrugineux } \\
\text { tropicaux, } \\
\text { Halomorphe, } \\
\text { Ferralitiques }\end{array}$ \\
\hline S. anguivi & Savanes boisées & Climat soudanien & $\begin{array}{l}\text { Sols ferrugineux } \\
\text { tropicaux }\end{array}$ \\
\hline S. terminale & Savanes arbustives & Climat soudanien & Sols rouge brun \\
\hline S. anomalum & $\begin{array}{l}\text { Savanes arbustives } \\
\text { Jachères }\end{array}$ & $\begin{array}{l}\text { Climat sahélo- } \\
\text { soudanien }\end{array}$ & $\begin{array}{l}\text { Sols : Bruns } \\
\text { subarides, } \\
\text { Ferrugineux tropicaux } \\
\text { Hydromorphe }\end{array}$ \\
\hline S. erianthum & $\begin{array}{l}\text { Savanes arbustives, } \\
\text { Jachères }\end{array}$ & Climat soudanien & $\begin{array}{l}\text { Sols ferrugineux } \\
\text { tropicaux }\end{array}$ \\
\hline S. cerasiferum & $\begin{array}{l}\text { Savanes boisées, } \\
\text { Savanes arbustives, } \\
\text { Jachères }\end{array}$ & $\begin{array}{l}\text { Climat sahélo- } \\
\text { soudanien et } \\
\text { soudanien }\end{array}$ & $\begin{array}{l}\text { Sols : Bruns } \\
\text { subarides, } \\
\text { Ferrugineux } \\
\text { tropicaux }\end{array}$ \\
\hline S. dasyphyllum & Forêts humides & $\begin{array}{l}\text { Climat soudano- } \\
\text { guinéen }\end{array}$ & $\begin{array}{l}\text { Sols : Bruns } \\
\text { subarides, } \\
\text { Ferrugineux } \\
\text { tropicaux, } \\
\text { Hydromorphes }\end{array}$ \\
\hline S. scabrum & Savanes arbustives & $\begin{array}{l}\text { Climat sahélo- } \\
\text { soudanien }\end{array}$ & $\begin{array}{l}\text { Sols ferrugineux } \\
\text { tropicaux }\end{array}$ \\
\hline S. sisymbriifolium & Forêts humides & $\begin{array}{l}\text { Climat soudano- } \\
\text { guinéen }\end{array}$ & Sols halomorphes \\
\hline S. seafortianum & Savanes arbustives & Climat soudanien & Sols brun subarides, \\
\hline S. forskalii & $\begin{array}{l}\text { Savanes arbustives } \\
\text { et steppes }\end{array}$ & Climat soudanien & $\begin{array}{l}\text { Sols : Rouge brun, } \\
\text { Ferrugineux } \\
\text { tropicaux }\end{array}$ \\
\hline
\end{tabular}




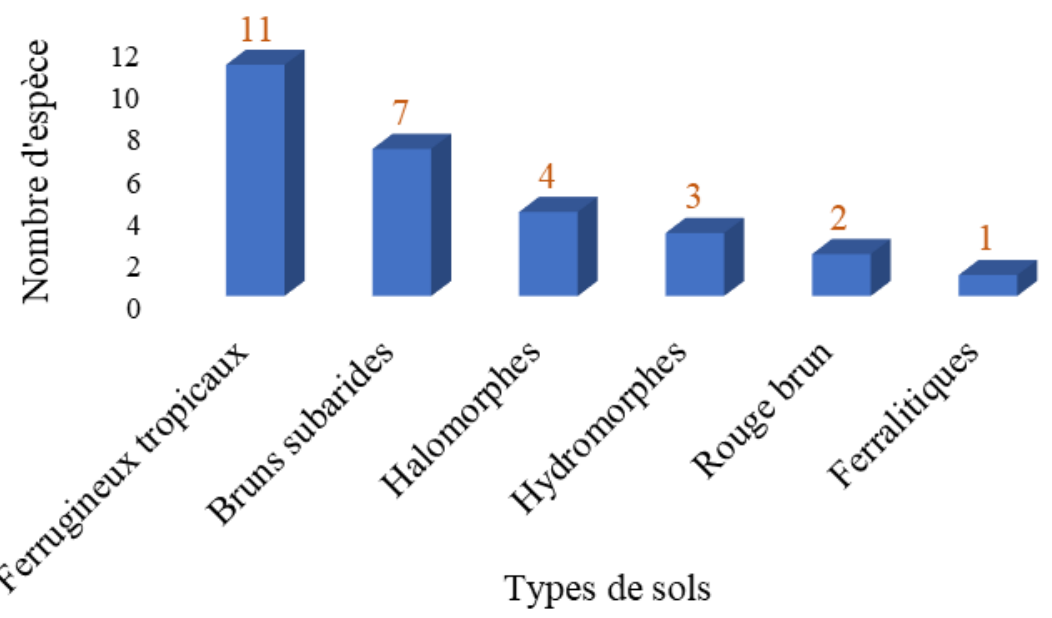

Figure 4 : Répartitions des espèces en fonction des sols

\section{Etude ethnobotanique}

Sur les 120 personnes enquêtées, 74,17\% sont de sexe masculin contre $25,83 \%$ de sexe féminin (Figure 5). La classe d'âge la plus représentative est celle des adultes $(82,5 \%)$ dont l'âge est compris entre 30 et 60 ans. Elle est suivie de celle des vieillards $(13,33 \%)$ de plus de 60 ans et enfin des jeunes $(4,17 \%)$ de moins de 30 ans (Figure 6). Ces informateurs sont d'ethnies diverses avec une forte dominance des Wolofs $(55 \%)$. Les sérères et les peulhs sont également bien représentés avec respectivement $20,66 \%$ et $18,18 \%$ tandis que les diolas sont faiblement représentés 6, 67\% (Figure 7). La plupart des enquêtés sont des agriculteurs avec $46,67 \%$ suivis des commerçants $24,16 \%$, des artisans 11,67\%, des éleveurs $10 \%$ et d'autres catégories socioprofessionnelles $7,5 \%$ (Figure 8 ). 


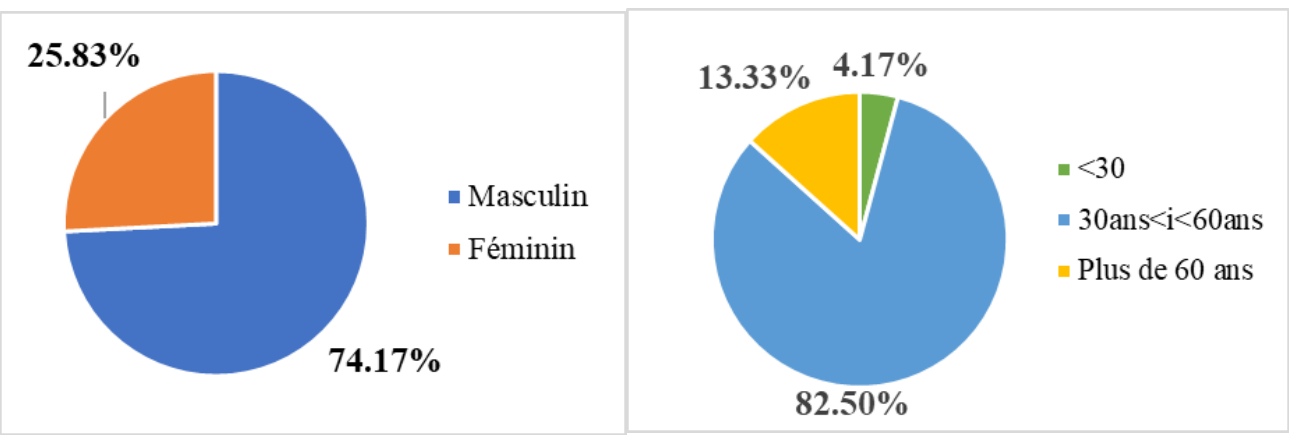

Figure 5 : Répartition des enquêtés selon le sexe

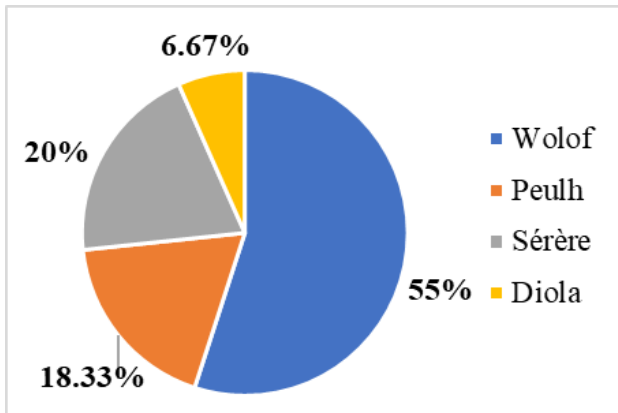

Figure 7 : Répartition des enquêtés selon les ethnies
Figure 6 : Répartition des enquêtés selon l'âge

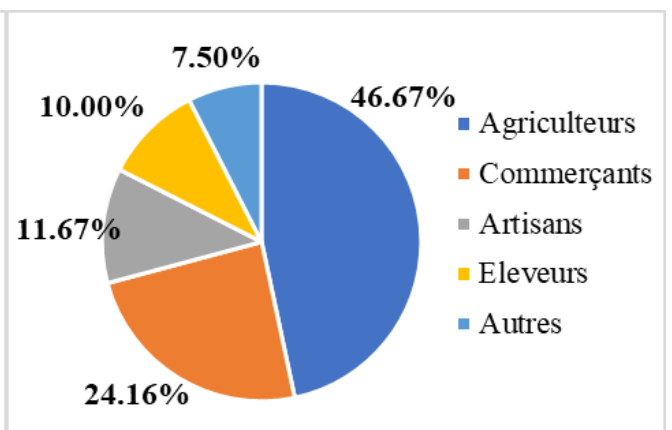

Figure 8 : Répartition des enquêtés selon les catégories socio-professionnelles

\section{Usages des espèces}

Le Tableau 4 résume les différents niveaux de connaissances et d'usages des espèces sauvages apparentées de légumes feuilles cultivées du genre Solanum rencontrées au Sénégal principalement dans les sites d'études.

Parmi les 15 espèces répertoriées, 14 sont exploitées par les populations. En effet, $S$. sisymbriifolium et $S$. nigrum sont les espèces les plus citées avec respectivement $23,13 \%$ et $20,65 \%$. Elles sont suivies de $S$. aculeatissimum $15,71 \%, S$. incanum $6,61 \%, S$. dasyphyllum $5,79 \%$ et $S$. torvum $5,79 \%$ qui sont les espèces moyennement citées par les enquêtés. Par ailleurs des espèces rarement citées sont notées avec moins de $5 \%$. Il s'agit de : S. anomalum 4,13\%, S. americanum 3,31\%, S. anguivi 3,31\%, S. seafortianum $3,31 \%$, S. scabrum $2,48 \%$, S. erianthum $2,48 \%$, S. cerasiferum $1,65 \%$ et $S$. terminale $1,65 \%$.

En ce qui concerne la nomenclature traditionnelle, 14 espèces ont des noms locaux. L'ethnie Wolof semble être plus engagée dans la dénomination des espèces car intervenant pour 12 espèces. Il est suivi des ethnies peulh et sérère avec 4 espèces chacune puis de l'ethnie Diola qui semble ne pas être engagé dans la dénomination de ces espèces. Les appellations locales peuvent être identiques d'une ethnie à l'autre. C'est l'exemple de : S. erianthum appelé «Kanni salade » chez les wolofs et les Sérères. De plus, au sein d'une même 
ethnie, on peut noter une diversité d'appellation de l'espèce. C'est le cas de $S$. americanum appelé «Ndary » ou encore «Badé kéllé » chez les peulhs.

L'exploitation des espèces concerne plusieurs domaines d'usages que sont : médicinal ; alimentation humaine ; culturel ; fourragère et artisanal.

- Dans le domaine de la médecine traditionnelle, 12 espèces sont utilisées. Il s'agit de: S. nigrum, $S$. aculeatissimum, $S$. incanum, $S$. dasyphyllum, S. torvum, S. anomalum, S. anguivi, S. seafortianum, $S$. scabrum, $S$. erianthum, $S$. cerasiferum, $S$. terminale. Six de ces espèces (S. nigrum, S. aculeatissimum, S. torvum, S. incanum, S. dasyphyllum et $S$. scabrum) sont utilisées pour soigner les douleurs de ventre et deux espèces ( $S$. anomalum et $S$. erianthum) interviennent dans le traitement des douleurs externes. Notons que $S$. cerasiferum est signalée dans la médecine animale.

- En ce qui concerne le domaine alimentaire, neuf espèces sont utilisées. Il s'agit de $S$. americanum, $S$. torvum, $S$. anomalum, $S$. scabrum, $S$. nigrum, S. incanum, S. terminale, S. erianthum et $S$. dasyphyllum.

- Pour le domaine de l'élevage (fourrage), six espèces ( $S$. aculeatissimum, $S$. anguivi, S. anomalum, S. incanum, S. terminale et S. nigrum) sont utilisées.

- Dans le domaine culturel, trois espèces (S. aculeatissimum, $S$. cerasiferum et $S$. sisymbriifolium) sont utilisées. Ces espèces sont utilisées pour se protéger contre les forces du mal (sorcellerie) et pour se purifier.

- Du point de vu artisanal, une espèce, S. seafortianum intervient pour la fabrication d'objet décoratif

Tableau 4 : Usages et noms vernaculaires des espèces sauvages apparentées de légumes feuilles cultivés du genre Solanum

\begin{tabular}{|c|c|c|c|}
\hline Espèces & $\begin{array}{c}\text { Nom vernaculaire } \\
\text { (Langue) }\end{array}$ & Usages (organes utilisés) & FC $(\%)$ \\
\hline $\begin{array}{l}\text { Solanum } \\
\text { sisymbriifoliu } \\
m\end{array}$ & Heum heum (Wolof) & $\begin{array}{l}\text { Culturel : utiliser pour la protection } \\
\text { contre le mal et les envoutements } \\
\text { (feuilles, fruits et racines) }\end{array}$ & 23,13 \\
\hline S. nigrum & $\begin{array}{l}\text { dagato foro (Foula), } \\
\text { basia bene (Malink, } \\
\text { Suludato } \\
\text { Mandingue), Gnoule ( } \\
\text { Wolof) }\end{array}$ & $\begin{array}{l}\text { Médicinal : utiliser pour traiter les } \\
\text { ulcères, les plaies chroniques, les } \\
\text { brûlures, (Feuilles et fruits) } \\
\text { Fourrage } \\
\text { Alimentaire : légume feuille }\end{array}$ & 20,65 \\
\hline $\begin{array}{l}\text { S. } \\
\text { aculeatissimu } \\
m\end{array}$ & $\begin{array}{l}\text { Botojan, hale mbeute } \\
\text { (Wolof), getingari } \\
\text { (Peulh) }\end{array}$ & $\begin{array}{l}\text { Médicinal : utiliser dans le traitement } \\
\text { de la constipation puis du rhumatisme } \\
\text { (feuilles) } \\
\text { Culturel : Purification, intervient dans } \\
\text { la lutte contre la sorcellerie (feuilles, } \\
\text { racines et fruits), } \\
\text { Fourrage }\end{array}$ & 15,71 \\
\hline
\end{tabular}




\begin{tabular}{|c|c|c|c|}
\hline S. incanum & $\begin{array}{l}\text { Itérénari (Toucouleur), } \\
\text { Ahatu (Wolof), Gitigari } \\
\text { (Peulh) }\end{array}$ & $\begin{array}{l}\text { Médicinal : utiliser pour soulager les : } \\
\text { maux d'estomac, maux de tête, règles } \\
\text { douloureuses, mal de dents (Fruits) } \\
\text { Fourrage } \\
\text { Alimentaire : les fruits }\end{array}$ & 6,61 \\
\hline S. dasyphyllum & $\begin{array}{l}\text { Kheubtal (Wolof et } \\
\text { Sèrère) }\end{array}$ & $\begin{array}{l}\text { Médicinal : Utiliser dans le traitement } \\
\text { des maux de ventre (racines, fruit) } \\
\text { Fourrage } \\
\text { Alimentaire : feuilles et fruits. }\end{array}$ & 5,79 \\
\hline S. torvum & $\begin{array}{l}\text { Pome, Batensé-all } \\
\text { (Wolof) }\end{array}$ & $\begin{array}{l}\text { Médicinal : utiliser pour soulager les : } \\
\text { douleurs de la gorge, maux } \\
\text { d'estomac, blessures (Feuilles et } \\
\text { racines) } \\
\text { Alimentaire: Fruit utilisés dans les } \\
\text { soupes }\end{array}$ & 5,79 \\
\hline S. anomalum & $\begin{array}{l}\text { nian-nian, tamate pithie } \\
\text { (Wolof et Peulh) }\end{array}$ & $\begin{array}{l}\text { Médicinal : utiliser pour soulager les } \\
\text { douleurs externes (feuilles) } \\
\text { Fourrage } \\
\text { Alimentaire : (feuilles et fruits) }\end{array}$ & 4,13 \\
\hline S. americanum & $\begin{array}{l}\text { Ndary, Badé } \quad \text { kelle } \\
\text { (Peulh) }\end{array}$ & $\begin{array}{l}\text { Alimentaire : consommer comme } \\
\text { légume (fruits et feuilles) }\end{array}$ & 3,31 \\
\hline S. anguivi & $\begin{array}{l}\text { Koukoudjè, } \\
\text { teumb (Wolof) }\end{array}$ & $\begin{array}{l}\text { Médicinal : utiliser dans la lutte } \\
\text { contre l'hypertension artérielle } \\
\text { (feuilles et fruits) } \\
\text { Fourrage }\end{array}$ & 3,31 \\
\hline $\begin{array}{l}\text { S. } \\
\text { seafortianum }\end{array}$ & Mbale (Wolof) & $\begin{array}{l}\text { Médicinal : utiliser pour soigner la } \\
\text { morsure du serpent (feuilles et fruit) } \\
\text { Artisanat : utiliser dans la fabrication } \\
\text { d'objet décoratif (feuilles) }\end{array}$ & 3,31 \\
\hline S. scabrum & Gnoule, Leung (Wolof) & $\begin{array}{l}\text { Médicinal : utiliser pour traiter la } \\
\text { diarrhée chez les enfants (Racines et } \\
\text { fruits) } \\
\text { Alimentaire : Les pousses } \\
\text { fraîches sont utilisées comme légume } \\
\text { feuilles }\end{array}$ & 2,48 \\
\hline S. erianthum & $\begin{array}{l}\text { Kanni salade (Wolof et } \\
\text { Sèrère) }\end{array}$ & $\begin{array}{l}\text { Médicinal : utiliser contre les maux de } \\
\text { tête, et les violentes douleurs } \\
\text { corporelles (Feuilles et fruits) } \\
\text { Alimentaire : Feuilles }\end{array}$ & 2,48 \\
\hline S. cerasiferum & $\begin{array}{l}\text { Batensé-bouki (Wolof et } \\
\text { Sèrère) }\end{array}$ & $\begin{array}{l}\text { Médicinal : utiliser pour soigner les } \\
\text { maladies parasitaires telles que la } \\
\text { coccidiose (Feuilles et racines) } \\
\text { Culturelle : Purgatif (Feuilles) }\end{array}$ & 1,65 \\
\hline S. terminale & $\begin{array}{l}\text { Hal-sagne (Wolof et } \\
\text { Sèrère) }\end{array}$ & $\begin{array}{l}\text { Médicinal : utiliser pour l'avortement } \\
\text { (racines) } \\
\text { Fourrage } \\
\text { Alimentaire : Feuilles et fruits }\end{array}$ & 1,65 \\
\hline S. forskalii & - & Non renseigné & - \\
\hline
\end{tabular}




\section{Les catégories d'usages}

L'exploitation des espèces sauvages apparentées des légumes feuilles cultivées du genre Solanum au Sénégal est assez variée au point de vu utilitaire (Figure 9). Les catégories d'usage les plus citées sont par ordre d'importance : médicinale $(47,87 \%)$, alimentaire $(27,66 \%)$, culturelle $(14,89 \%)$, fourragère $(8,51 \%)$ et artisanale $(1,06 \%)$.

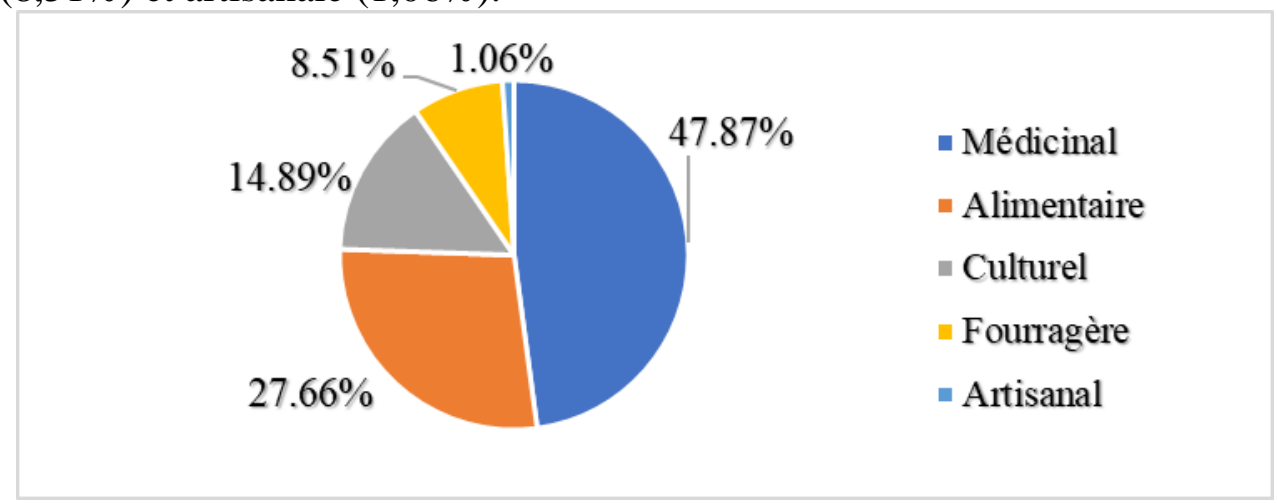

Figure 9 : Spectre des différents usages des espèces

Pour ce qui est de la diversité d'usage, la Figure 10 montre trois groupe d'espèces en fonction de leur intervention dans les différents besoins :

- Les espèces ayant une diversité d'usages élevée (intervenant dans trois catégories d'usage). Il s'agit de $S$. aculeatissimum, $S$. incanum, $S$. terminale, $S$. anomalum et $S$. nigrum

- Les espèces ayant une diversité d'usages moyenne (intervenant dans deux catégories d'usage). Il s'agit de $S$. dasyphyllum, $S$. anguivi, $S$. erianthum, $S$. cerasiferum, $S . \quad$ scabrum, $S$. sisymbriifolium, $S$. seafortianum, et $S$. torvum.

- Les espèces à diversité d'usages faible (intervenant dans une catégories d'usage). Il s'agit de S. americanum

Pour ce qui est des catégories d'usage, la Figure 10 montre que, la médecine est l'usage le plus fréquent car concernant 12 espèces. Elle est suivie des catégories alimentaire ( 9 espèces), fourragère ( 6 espèces), culturelle (4 espèces) et artisanale (1 espèces) 


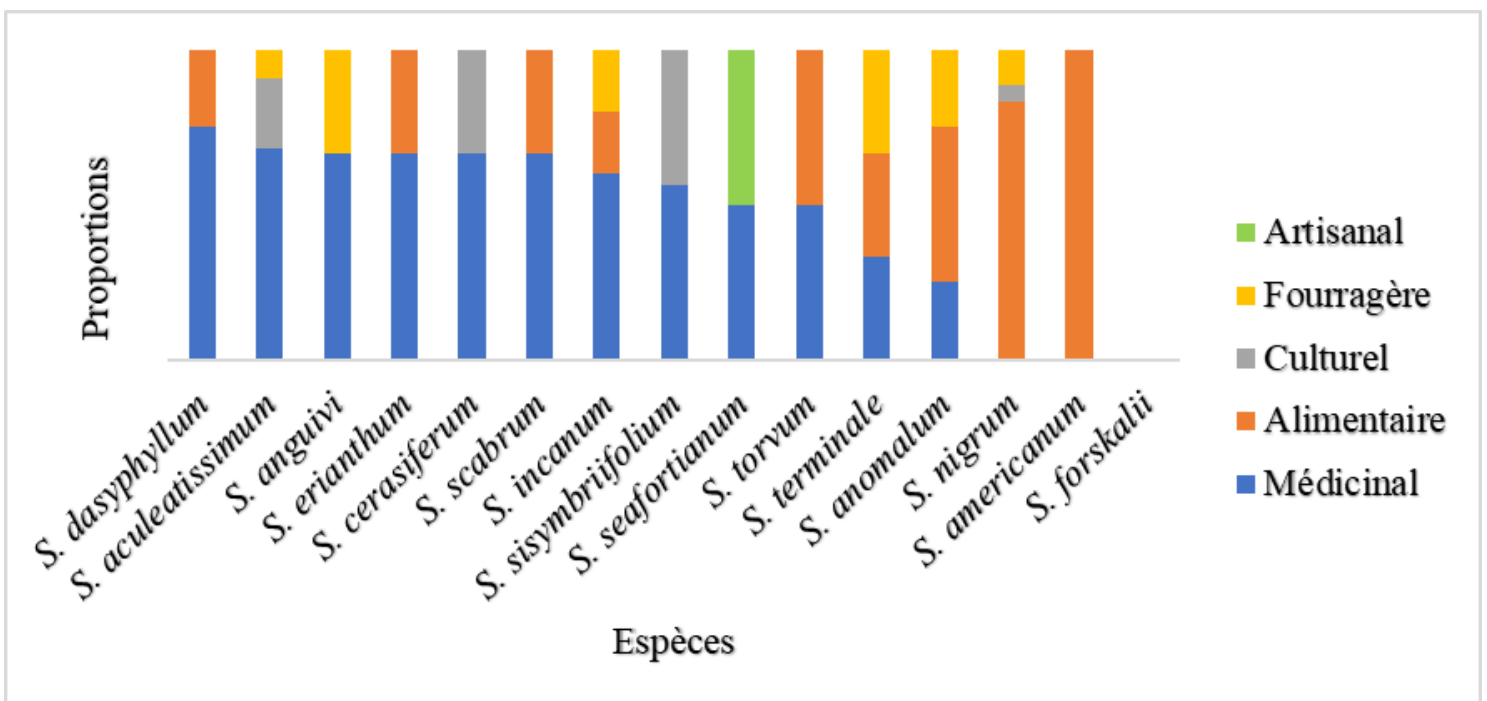

Figure 10 : Importance relative des catégories d'usage des quinze (15) espèces sauvages apparentées des légumes feuilles du genre Solanum retrouvées au Sénégal

\section{Usages des organes}

Les fréquences d'utilisation des organes des espèces du genre Solanum sont mentionnées sur la Figure 11. La feuille $(43,36 \%)$ est l'organe le plus exploité, suivie de la racine $(30,97 \%)$, du fruit $(24,78 \%)$ et la tige $(0,88 \%)$.

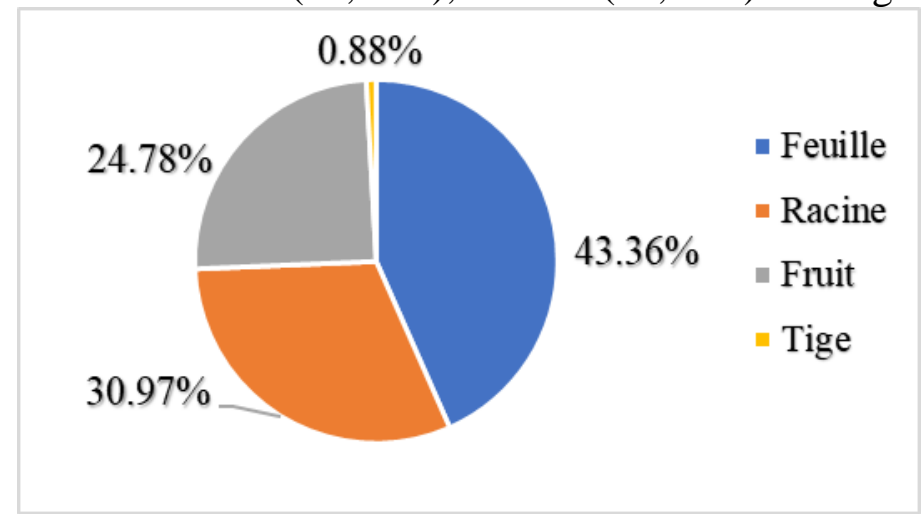

Figure 11 : Spectre des différents organes des espèces

L'analyse de la Figure 12 révèle l'utilisation de quatre organes des espèces étudiées. Celle-ci varie en fonction des espèces.

- Les fruits de toutes les espèces étudiées sont exploités ;

- Les feuilles de treize espèces (13), excepté celles de Solanum cerasiferum, sont utilisées ;

- La racine de sept (7) espèces suivantes : S. terminale, S. dasyphyllum, $S$. aculeatissimum, $S$. scabrum, $S$. torvum, $S$. sisymbriifolium et $S$. cerasiferum est utilisée ; 
- $\quad$ La tige d'une seule espèce, $S$. sisymbriifolium est utilisée.

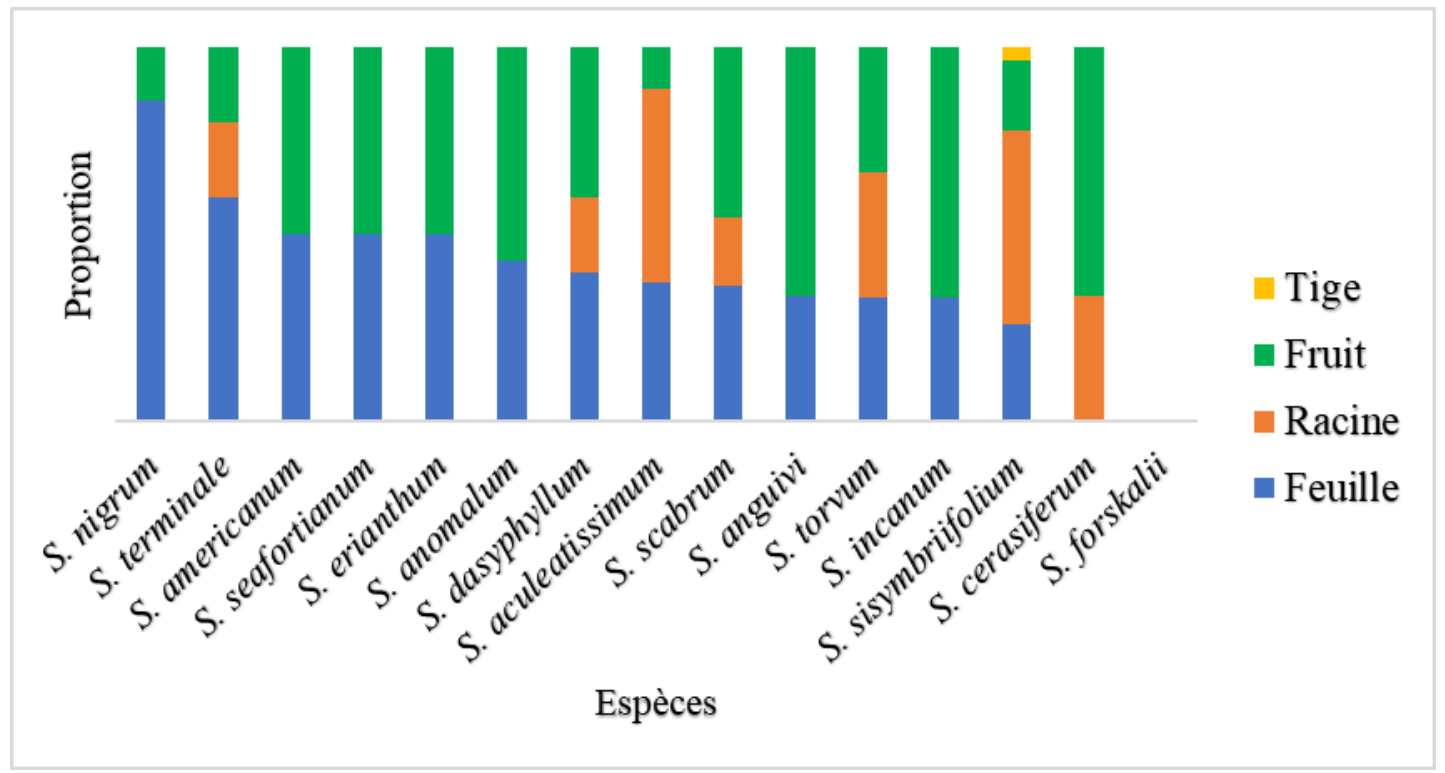

Figure 12 : Importance relative des organes utilisés des espèces sauvages apparentées des légumes feuilles cultivées du genre Solanum au Sénégal

\section{Connaissance des espèces dans les sites d'étude}

L'Analyse Factorielle des Correspondances est réalisée pour identifier les espèces sauvages apparentées de légumes feuilles cultivés les plus citées par région (Dakar, Saint Louis et Thiès) (Figure 13). L'axe 1 horizontal porte $76.04 \%$ de l'inertie totale et l'axe 2 vertical porte $23.96 \%$ de l'inertie totale, ces deux axes cumulent la totalité de l'inertie du tableau des données initiales. Le graphe de l'AFC permet de distinguer trois (03) groupes. Le groupe A est isolé suivant l'axe 1 dans les abscisses positives et les groupes $\mathrm{B}$ et $\mathrm{C}$ par l'axe 2 respectivement dans les ordonnées positive et négative (Figure 13).

Le groupe A caractérise les espèces dont l'occurrence est significativement plus importante dans la région de Dakar notamment «SOLNI » avec une contribution de 65,19 (Tableau 5). Le groupe B qui est caractérisé par les espèces : S. americanum $(0.81), S$. sisymbriifolium $(16,62)$ et $S$. aculeatissimum $(0,05)$ (Tableau 5) dont l'occurrence est plus importante dans la région de Saint-Louis «SOLSI » s'oppose au groupe C caractérisé par des espèces : $S$. dasyphyllum $(3,18)$ et $S$. erianthum $(1,49)$ (Tableau 5$)$ dont l'occurrence est plus importante dans la région de Thiès «SOLDA ».

Les huit (8) autres espèces représentent des intermédiaires entre les trois groupes. Ces espèces n'ont pas montré une affection particulière pour une région. Il faut néanmoins distinguer deux (2) catégories d'espèces. 
- Les espèces dont la connaissance est réduite à deux régions. Il s'agit de $S$. scabrum, $S$. terminale, $S$. anguivi (Saint Louis et Thiès) et $S$. cerasiferum (Thiès et Dakar).

- Les espèces connues dans les trois régions avec des contributions rapprochées. Il s'agit de $S$. anomalum, S. incanum, S. seafortianum et S. torvum.

De manière générale, cette analyse ne révèle pas de corrélation véritable entre les localités et les espèces étudiées. Ainsi, l'influence du micro climat de la forêt classée de Mbao puis la forte présence d'activité agricole (maraichage) dans cette zone permettrait d'expliquer l'abondance de $S$. nigrum dans la région de Dakar. 
Tableau 5 : Contribution de chaque espèce

\begin{tabular}{|c|c|c|c|c|c|c|c|}
\hline & Iner*1000 & Dim.1 & $\mathrm{Ctr}$ & $\cos 2$ & Dim.2 & ctr & $\cos 2$ \\
\hline SOLAC & 6.471 & -0.029 & 0.056 & 0.020 & 0.202 & 8.680 & 0.980 \\
\hline SOLAN & 7.164 & -0.502 & 2.943 & 0.953 & -0.112 & 0.465 & 0.047 \\
\hline SOLANO & 1.270 & -0.161 & 0.529 & 0.965 & -0.031 & 0.061 & 0.035 \\
\hline SOLDA & 30.838 & -0.370 & 3.188 & 0.240 & -0.659 & 32.096 & 0.760 \\
\hline SOLIN & 2.471 & 0.153 & 1.018 & 0.956 & -0.033 & 0.150 & 0.044 \\
\hline SOLAM & 27.840 & -0.237 & 0.816 & 0.068 & 0.876 & 35.519 & 0.932 \\
\hline SOLSI & 39.948 & -0.404 & 16.629 & 0.965 & 0.077 & 1.901 & 0.035 \\
\hline SOLTE & 3.582 & -0.502 & 1.471 & 0.953 & -0.112 & 0.233 & 0.047 \\
\hline SOLSE & 2.297 & 0.161 & 0.304 & 0.307 & -0.243 & 2.180 & 0.693 \\
\hline SOLTO & 3.557 & -0.237 & 1.472 & 0.959 & -0.049 & 0.198 & 0.041 \\
\hline SOLNI & 151,169 & 0.92760895 & 65.19094803 & 0.99994571 & 0.006834858 & 0.01123403 & $5.428822 \mathrm{e}-05$ \\
\hline SOLER & 8,075 & -0.41401225 & 1.49841363 & 0.43023360 & -0.476441343 & 6.29858834 & $5.697664 \mathrm{e}-01$ \\
\hline SOLCE & 10,308 & 0.47123284 & 1.94122753 & 0.34409641 & -0.650602142 & 11.74506223 & $6.559036 \mathrm{e}-01$ \\
\hline SOLSC & 7,163 & -0.50247435 & 2.94287539 & 0.95257793 & -0.112112464 & 0.46501919 & $4.742207 \mathrm{e}-02$ \\
\hline
\end{tabular}

$\mathrm{SOLAC}=$ S. aculeatissimum, $\mathrm{SOLAN}=$ S. anomalum, $\mathrm{SOLANO}=S$. anomalum, $\mathrm{SOLDA}=$ S. dasyphyllum, $\mathrm{SOLIN}=S$. incanum, $\mathrm{SOLAM}=S$. Americanum, $\mathrm{SOLSI}=$ S. sisymbriifolium, $\mathrm{SOLTE}=$ S. terminale, $\mathrm{SOLSE}=$ S. seafortianum $\mathrm{SOLTO}=$ S. torvum, $\mathrm{SOLNI}=S$. nigrum, $\mathrm{SOLER}=$ S. erianthum, $\mathrm{SOLCE}=S$. cerasiferum, $\mathrm{SOLSC}=S$. scabrum. 


\section{CA factor map}

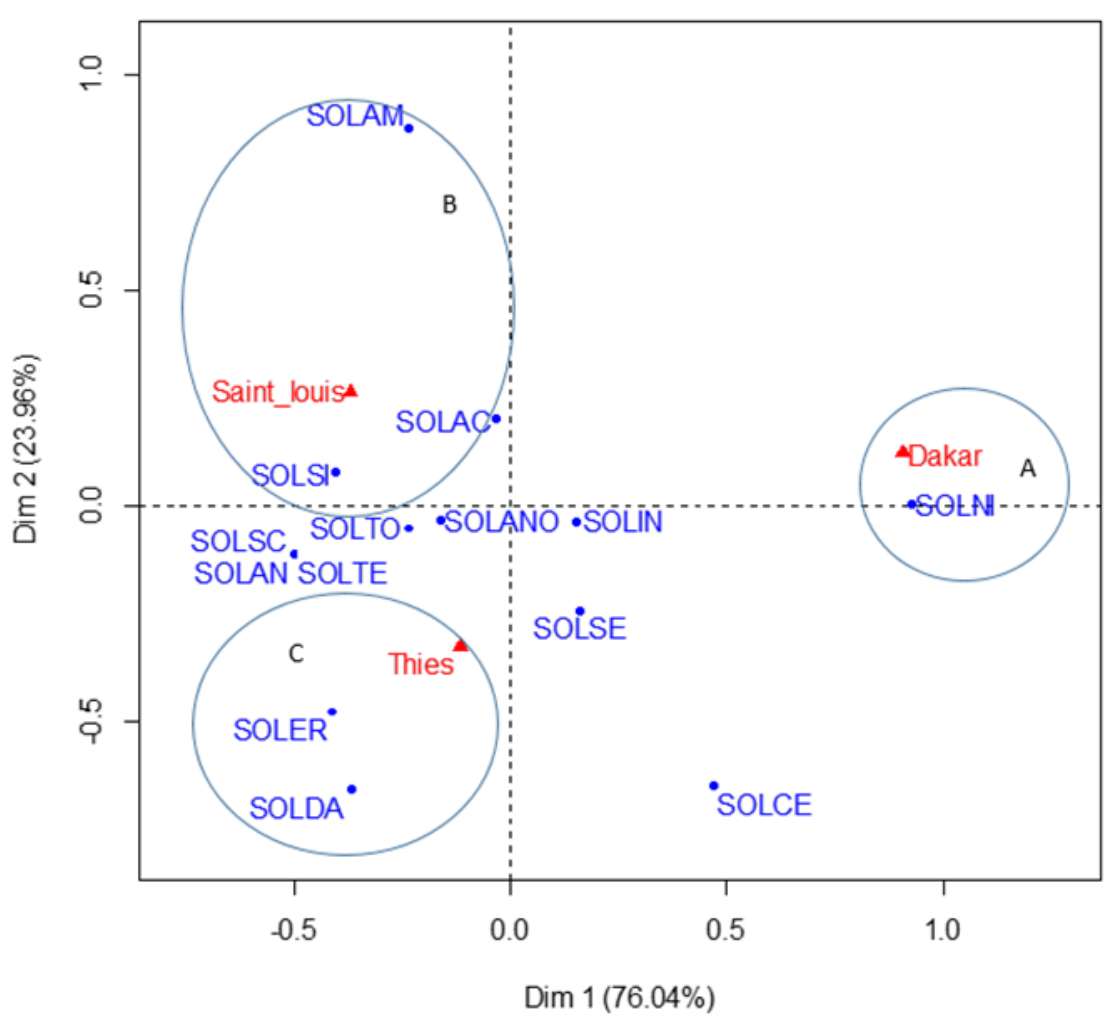

Figure 13 : Analyse Factorielle des Correspondances (AFC) croisant la variable Espèce (15 Espèces) et la variable région (3 régions) dans le plan factoriel dim1xdim2.

\section{Discussion}

\section{Chorologie et écologie}

De nombreuses études ont montré que les espèces du genre Solanum se rencontrent en Amérique du Sud ; ce qui suppose que leurs plus grande diversité se trouve dans cette partie du monde (Hunziker, 1979; Samuels, 2015). Cependant, elles présentent une large distribution en Afrique et en Asie (Bukenya \& Carasco, 1995). Sur le continent Africain, elles sont retrouvées à l'état sauvage ou domestiqué dans plusieurs pays (Manoko et al., 2008 ; Edmonds \& Chweya, 1997 ; Fontem \& Schippers, 2004). C'est le cas de l'Ouganda où Bukenya \& Carasco (1995) ont inventorié 41 espèces appartenant au genre Solanum et du Nigéria où Gbile \& Adesina (1988) ont dénombré environs 25 espèces. Au Sénégal, plusieurs documents scientifiques tels que les flores illustrées du Sénégal (Berhaut, 1967; 1988) confirment la présence des espèces étudiées dans la zone agroécologique des Niayes.

Les résultats de la répartition géographiques ont révélé la présence de onze (11) espèces sauvages apparentées aux légumes feuilles cultivés du genre Solanum dans la zone agro-écologique des Niayes qui est une zone côtière 
humide constituée d'écosystèmes fragiles, riches en biodiversité (Ndao, 2012).

En effet, ces espèces affectionnent mieux les milieux humides et elles sont retrouvées sur divers types de sol dans les milieux ouvert (Bukenya \& Ziraba, 1996). Cela expliquerais donc leurs forte présence dans la zone des Niayes car cette dernière se singularise du reste du pays par l'influence des vents marins forts et relativement constants toute l'année (Fall, 1986; Faye et al., 2014). L'humidité élevée du sol de la zone et les températures adoucies par les vents marins ont favorisé l'installation d'espèces sub-guinéennes dont l'aire de répartition naturelle est couverte par les isohyètes $1200 \mathrm{~mm}$. De plus, les Niayes sont des espaces inondables à caractère semi naturel avec un degré de diversité biologique exceptionnel (Ndao, 2012). Cependant, le faible nombre d'espèces sauvages apparentées de légumes feuilles cultivées du genre Solanum signalés dans la zone de la Casamance qui bénéficie d'un climat subguinéen ne doit pas être considéré comme définitive. Il est certain que cette zone a été peu explorée. Ces mêmes observations sur le faible nombre d'espèce identifier dans cette zone ont été faites sur la chorologie du genre Ficus dans les travaux de Diop et al. (2012). En effet, l'utilisation par l'homme de la plupart de ces espèces pour des raisons diverses (alimentaire, nutritionnel, médicinal, ornementale ...) couplée à la présence de la forêt classée de Mbao constitue un atout majeur pour la dissémination des graines de ces espèces par les populations animales et humaines dans cette zone. Par ailleurs, la forte présence des espèces sur des sols ferrugineux tropicaux s'explique par la prédominance de ces derniers au Sénégal et notamment dans la zone des Niayes (Touré \& Seck, 2005).

\section{Etude ethnobotanique}

La connaissance et l'exploitation des espèces sauvages apparentées sont les preuves avérées de leurs utilités dans les relations entre l'homme et son environnement (Kimpouni et al., 2005). Les espèces sauvages apparentées du genre Solanum étudiées sont connues par les quatre ethnies majoritaires de la zone d'étude. Toutefois, on reconnait une différence d'appellation entre les ethnies et même au sein d'un même groupe. Cette variabilité nominale et même polyonymie relève d'une aberration de la transmission orale d'une génération à une autre comme le soulignent Ta Bi et al. (2016); Djihounouck et al. (2019). Les résultats montrent que les espèces du genre Solanum sont majoritairement à usage médicinale et alimentaire. Sur le plan médicinal, les populations locales enquêtées reconnaissent que des organes (feuilles, fruits et racines) de plusieurs espèces spontanées du genre Solanum donnent une lotion très efficace pour le traitement de diverses infections internes et externes. Ce traitement traditionnel corrobore les résultats obtenus par Ogunwande et al. (2013) qui ont montré que les principes actifs tels que la solanidine et d'autres 
stéroïdes extraites des racines et des feuilles de certaines de ces espèces (Solanum torvum et Solanum nigrum) entrent dans la fabrication de nombreux produits pharmaceutiques. De même, Gbile \& Adesina (1988) ont signalé l'usage des espèces du genre Solanum dans la thérapie cardiovasculaire comme agents anti-inflammatoires et anticancéreux. Ces propriétés intrinsèques des espèces justifient le fait que beaucoup d'entre elles sont utilisées comme remèdes contre le rhume, la fièvre, les douleurs abdominales, les céphalées-migraines, les règles douloureuses, les maux de dents...etc. En Afrique, le rôle important des espèces spontanées apparentées de légumes feuilles cultivées du genre Solanum dans l'alimentation humaine est démontré dans de nombreux travaux de recherche tels que ceux de Ogundajo et al. (2013) ; Adjatin et al. (2019). Ils seraient donc très utile pour les programmes d'amélioration génétique des espèces cultivées et pourraient aussi fortement servir de compléments nutritionnels aux espèces exotiques (Musinguzi et al., 2007; Ouattara et al., 2016). Cependant, S. nigrum, jadis bien consommé selon les populations locales, est aujourd'hui de plus en plus considéré comme un aliment des personnes démunies puisqu'elle est spontanée et abonde dans la nature. Cette vision péjorative et complexée des populations sur les végétaux spontanés a été déjà signalée par Okouango et al. (2015) qui ont montré qu'en Afrique, les légumes feuilles sauvages sont généralement négligés par les consommateurs en raison des idées péjoratives qui leur sont attribuées comme indicatrices de pauvreté ou de rang social arriéré.

L'étude a aussi montré que les parties les plus utilisées de ces espèces sont les feuilles à l'état naturel ou cuites, suivi des racines et des fruits qui sont consommés directement et /ou utilisés dans divers traitements médicinaux ou des rituels de purification et de protection. Certaines espèces telles que $S$. sisymbriifolium et $S$. aculeatissimum interviennent dans ces rituels et permettraient de résister aux attaques mystiques. Ces résultats, corroborent ceux de Gbile \& Adesina (1988) qui ont montré que les fruits et les feuilles de certaines espèces ( $S$. dasyphyllum, S. scabrum, S. nigrum ...) sont mangés comme légumes et utilisés dans la médecine traditionnelle. De plus, les feuilles spontanées constituent un complément alimentaire important pour les populations des zones sahélo-soudaniennes qui n'ont pas la possibilité de se procurer fréquemment de protéines animales (Guèye \& Diouf, 2007).

L'Analyse Factorielle des Correspondances réalisée révèle que la connaissance des espèces sauvages apparentées aux légumes feuilles du genre Solanum répertoriées varie d'une localité à une autre. Ainsi, la différenciation des trois groupes $\mathrm{A}, \mathrm{B}$ et $\mathrm{C}$ peut être expliquée par la variation des préférences alimentaires, médicinales et culturelles des ethnies présentes dans la zone des Niayes ainsi que leur niveau de vie. Afin d'éviter la perte de ces espèces d'importance, les structures en charge de la protection des végétaux devraient procéder à des sensibilisations de la population locale sur leurs utilités, 
notamment dans les milieux ou leurs diversités sont relativement faible mais aussi identifier des milieux pouvant servir de zone de conservation in situ des espèces. L'introduction des espèces dans les jardins botaniques pourrait également constituer un atout majeur pour leurs protections.

\section{Conclusion}

Le présent travail contribue à une meilleure connaissance de la répartition géographique, de l'écologie et des usages des espèces sauvages apparentées de légumes feuilles cultivés au Sénégal. Toutes les espèces étudiées sont présentes en Afrique tropicale bien qu'elles semblent avoir leur plus grande diversité en Amérique. Au Sénégal, l'étude montre que les espèces sont présentes dans toutes les zones agro écologique et donc occupent des habitats très variés. Toutefois la plupart d'entre elles se trouvent au nord du pays plus précisément dans la zone des Niayes où elles bénéficient des conditions environnementales relativement favorables. Les différents types de sols et de formations végétales occupés par ces espèces attestent leur forte capacité d'adaptation. Les investigations ethnobotaniques ont permis de notées que les espèces sont bien connues de la population avec des noms locaux attribués à chacune. Cette connaissance liée aux espèces explique leur exploitation dans plusieurs besoins avec une préférence dans le domaine médicinale et alimentaire. Ainsi, l'organe le plus recherché dans ces usages reste la feuille pour ces vertus thérapeutiques. Donc ce qui nous revient à envisager que la population avait une connaissance traditionnelle sur l'importance de cet organe dans la production des molécules bioactives. Etant donné l'intérêt suscité aux espèces sauvages apparentées du genre Solanum par les populations, nous envisageons de réaliser un inventaire au niveau national afin de proposer des stratégies de gestion de celles les plus vulnérables.

\section{Conflit d'intérêt}

Les auteurs déclarent n'avoir aucun conflit d'intérêt dans ce manuscrit.

\section{Contribution des auteurs.}

C. S. A. a effectué les enquêtes de terrain, dépouillé et traité les données puis initié la rédaction de cet article.

N. D. et J. D. sont des Botanistes, ils ont participé à la collecte des données et à l'identification des espèces.

G. M. K., Y. D et A. N. ont lu le protocole et amélioré le manuscrit rédigé.

D. D., M. S. M., A. E. A., et K. N. ont supervisé le travail et amélioré le manuscrit.

Tous les auteurs ont lu et approuvé le manuscrit définitif. 


\section{References:}

1. Adam, J.G. (1961) - Contribution à l'étude de la flore et de la végétation de l'Afrique occidentale. La basse Casamance (Sénégal). Première partie. Bull. IFAN, t. XXIII, Sér. A, n4, pp. 911-993.

2. Adam, J.G. (1962) - Contribution à l'étude de la flore et de la végétation de l'Afrique occidentale. La basse Casamance (Sénégal). Deuxième partie. Bull. IFAN, t. XXIV, Sér. A, n 1, pp. 116-153.

3. Adam, J.G. (1970) - Noms vernaculaires des plantes du Sénégal. Journal d'Agriculture tropicale et de botanique appliquée, T. 17 (7-89), $112 \mathrm{p}$.

4. Adam, J.G. (1981) - Flore descriptive des Monts Nimba. (Cote d'Ivoire, Guinée, Liberia). Vol. 5. Paris : CNRS, pp. 1587-2051.

5. Adjatin, A., Adjahossou, N., Vodouhè, S., Dandjinou, P., Aboudou, R., Yedomohan, H., \& Dansi, A. (2019) - Caractéristiques ethnobotaniques et pratiques culturales de Solanum. scabrum Mill., légume-feuilles en domestication dans le département du Plateau au Sud Bénin. International Journal of Innovation and Applied Studies, 27, pp. 910-923.

6. Akpavi, S., Woegan A.Y., Dourma, M., Tozo K., Batawila K., Wala K., Gbogbo, K.A., Kanda, M., Kossi-Titrikou, K. \& de Foucault, B. (2011) - Que sont devenues les plantes autrefois consommées par les divers groupes ethnoculturels du Togo ? Agronomie Africaine, 23, pp. 147-160.

7. Alsherbiny, M.A., Ezzat, S.M., Elsakhawy F.S., Kamel, G.M. \& Abdel-Kawy, M.A. (2015) - Impact of certain Solanum speciess natural products as potent cytotoxic and anti-Inflammatory agents. $J$. Med. Plants Res, 9, pp. 779-786.

8. Alves, R.R. \& Alves, H.N. (2011) - The faunal drugstore: Animalbased remedies used in traditional medicines in Latin America. Journal of ethnobiology and ethnomedicine, 7, 9p.

9. Berhaut, J. (1967) - Flore du Sénégal plus complète avec les forêts humides de la Casamance. Ed. Clairafrique, Dakar 485p.

10. Berhaut, J. (1979) - Flore illustrée du Sénégal. Gouvernement du Sénégal, Ministère du développement rural, Direction des Direction des Eaux et Forêt. Dakar - Sénégal. T. 1 : pp. 406-408.

11. Berhaut, J. (1988) - Flore illustrée du Sénégal. Gouvernement du Sénégal, Ministère du développement rural, Direction des Direction des Eaux et Forêt. Dakar - Sénégal. T. 1 : pp. 406-408.

12. Bukenya, Z. R., \& Carasco, J. F. (1995) - Solanum (Solanaceae) in Uganda Bothalia, 25(1), 43-59.

13. Bukenya-Ziraba, R. (1996) - Uses, chromosome number and distribution of Solanum species in Uganda. In the Biodiversity of 

African Plants (pp. 33-37). Springer, Dordrecht. https://doi.org/10.1007/978-94-009-0285-5_5.

14. Clay, E. (2002) - Food security: concepts and measurement. Trade reforms and food security: Conceptualising the linkages, pp. 25-34.

15. Diallo, A., Guisse, A., Ngom, Faye, M., Sapadoum, G. (2009) Variabilité floristique de la végétation herbacée de la Niaye de Pikine au Sénégal. Rev. Ecol. Terre Vie, 64 : 123- 133.

16. Diop, D., Mbaye, M.S., Kane, A., Sambou, B. \& Noba K. (2012) Chorologie, écologie et ethnobotanique de certains Ficus sp. L. (Moraceae) au Sénégal. BASE. 16(1), 13-24

17. Djihounouck, Y., Diop, D., Bassène, C., Mbaye, M., Diop, R., Faye, B., \& Noba, K. (2019) - Étude ethno-taxonomique des espèces fruitières spontanées comestibles chez l'ethnie Diola d'Oussouye (Sénégal). Vertigo : la revue électronique en sciences de l'environnement, 19(1). URI : https://id.erudit.org/iderudit/1065434ar.

18. Dossou, M.E., Houessou, G.L., Lougbégnon, O.T., Tenté, A.H.B. \& Codjia, J.T.C. (2012) -Ethnobotanical study of ligneous species in Agonvè swampy forest and surrounding area in Benin. Tropicultura, 30, pp. 41-48.

19. Edmonds, JM \& Chweya, JA. (1997) Black nightshades. Solanum nigrum L. and related species. Institute of Plant Genetics and Crop Plant Research Gatersleben/International Plant Genetic Reources Institute, Rome, Italy, pp 113

20. Fah, L., Klotoé, J.R., Dougnon, V., Koudokpon, H., Fanou, V.B.A., Dandjesso, C. \& Loko F. (2013) - Etude ethnobotanique des plantes utilisées dans le traitement du diabète chez la femme enceinte à Cotonou et Abomey-Calali (Benin). Journal of Animals et plant Sciences 18(1) : 2647-2658.

21. Fall, M. (1986) - Environnement sédimentaire quaternaires et actuels des tourbières des Niayes de la grande côte du Sénégal. Thèse de docotorat Université de Dakar, 130 p.

22. Faye, E., Dieng, H., Bogaert, J. \& Lejoly, J. (2014) - Dynamique de la flore et de la végétation des Niayes et du Bassin arachidier au Sénégal. Journal of Agriculture and Environment for International Development (JAEID), 108, 191-206. DOI: 10.12895/jaeid.20142.240

23. Fontem, DA. \& Schippers, RR (2004) - Solanum scabrum Mill. In: Grubben GJH, Denton OA (eds) Plant Resources of Tropical Africa 2. Vegetables, PROTA Foundation Wageningen/CTAWageningen/ Backhuys Publishers, Leiden, Netherlands, pp 493-498

24. Frodin, D.G. (2004) - History and concepts of big plant genera. Taxon, 53, pp. 753-776. 
25. Gbile, Z.O. \& Adesina S.K. (1988) - Nigerian Solanum species of economic importance. Annals of the Missouri Botanical Garden, 862865.

26. Guèye, M. \& Diouf M. (2007) - Traditional leafy vegetables in senegal: Diversity and medicinal uses. African Journal of Traditional, Complementary and Alternative Medicines, 4, pp. 469-475.

27. Hunter, D \& Heywood, V. (eds.). (2011) - Crop Wild Relatives: A Manual of In Situ Conservation. Earthscan, London and Washington, DC. $414 \mathrm{pp}$.

28. Hunziker, A.T. (1979) - South American Solanaceae: a synoptic survey. In: Linnean Society symposium series

29. Hutchinson J, Dalziel JM (1958). Flora of Tropical West Africa vol.

1(2) Crown agents. London. Invest. New Drugs; 29(4): 583-592

30. Jain, R., Sharma, A., Gupta, S., Sarethy, I.P. \& Gabrani, R. (2011) Solanum nigrum: current perspectives on therapeutic properties. Altern Med Rev, 16, pp. 78-85.

31. Kerharo, J. \& Adam, J.G. (1974) - La pharmacopée sénégalaise traditionnelle - Plantes médicinales et toxiques, Vigot Frères, Paris, France.

32. Kimpouni V, Mamboueni JC, Lenga-Sacadura M-Y, Nsika Mikoko E. 2017 - Recipes and Treatments in Traditional Herbal Medicine to the Kaamba Community of Madingou, Congo. European Journal of Medicinal Plants 20:1-13, doi10.9734/EJMP/2017/35096.

33. Lebrun, J.P., A.L. Stork, 1991, 1992, 1995, 1997 - Énumération des plantes à fleurs d'Afrique tropicale Genève : Conservatoire et jardin botanique de Genève, pp. 1-4.

34. Manoko, M. L., van den Berg, R. G., Feron, R. M., van der Weerden, G. M., \& Mariani, C. (2008) - Genetic diversity of the African hexaploid species Solanum scabrum Mill. and Solanum nigrum L. (Solanaceae). Genetic Resources and Crop Evolution, 55(3), 409-418. https://doi.org/10.1007/s10722-007-9248-Z

35. Maxted, N., Ford-Lloyd B.V., Jury S., Kell S. \& Scholten M. (2006) Towards a definition of a crop wild relative. Biodiversity \& Conservation, 15, pp. 2673-2685. https://doi.org/10.1007/s10531005-5409-6

36. Maxted, N. \& Kell N. S. (2009) - Establishment of a global network for the in-situ conservation of crop wild relatives: status and needs. FAO consultancy report, FAO, Rome, pp 1-265.

37. Mbow, C. (2009) - Potentiel et dynamique des stocks de carbone des savanes soudaniennes et soudano-guinéennes du Sénégal. Thèse de Doctorat d'Etat, Université Cheikh Anta Diop de Dakar, 319 p. 
38. MEPN (1997) - Rapport National Biodiversité. Ministère de l'Environnement et de la Protection de la Nature (MEPN), Dakar, Sénégal. Available at: https://www.cbd.int/doc/world/sn/sn-nr-01fr.pdf

39. Musinguzi, E., Kikafunda, J.K. \& Kiremire, B.T. (2007) - Promoting indigenous wild edible fruits to complement roots and tuber crops in alleviating vitamin A deficiencies in Uganda. Proceedings of the 13th ISTRC Symposium, pp. 763-769.

40. Ndao, M. (2012) - Dynamiques et gestions environnementales de 1970 à 2010 des zones humides au Sénégal : étude de l'occupation du sol par télédétection des Niayes avec Djiddah Thiaroye Kao (à Dakar), Mboro (à Thiès) et Saint-Louis, Thèse de doctorat, université de Toulouse (France), université Gaston Berger de St-Louis, 370 p.

41. Ngom, A., Mbaye, M. S., Barnaud, A., Kane, A., Ba, N., Gueye, M., Diop, B. M. \& Noba, K. (2016) - Révision du genre Digitaria Haller (Poaceae) au Sénégal : proposition d'une clé de détermination pour une meilleure identification des espèces. International Journal of Biological and Chemical Sciences, 10(1), 58-86.

42. Obute, G.C., Ndukwu B.C. \& Okoli B.E. (2006) - Cytogenetic studies on some Nigerian species of Solanum L. (Solanaceae). African journal of biotechnology, 5(9):689-692.

43. Ogundajo, A. L., Oladosu, I.A., Ogunwande, I.A., Flamini G. \& Owolabi, M.S. (2013)- "Study on volatile constituents of Solanum nigrum var. viriginicum from Nigeria." pp. 94-98. http://hdl.handle.net/11568/159290

44. Ogunwande I.A., Avoseh N.O., Flamini G., Hassan A.S., Ogunmoye A.O., Ogunsanwo A.O., Yusuf K.O., Kelechi A.O., Tiamiyu Z.A., and Tabowei G.O. Essential oils from the leaves of six medicinal plants of Nigeria. Natural Product Communications 2013; 8: 243-248.

45. Okouango, I., Michel, E., Moutsamboté J.M., Vital M. \& François M. (2015) - Évaluation de la consommation et de la composition nutritionnelle des légumes-feuilles de Phytolacca dodecandra L'Herit consommés par les populations originaires des districts d'Owando et de Makoua. Journal of Animal \&Plant Sciences, 27, pp. 4207-4218.

46. Ouattara, N.D., Gaille, E., Stauffer F.W. \& Bakayoko A. (2016) Diversité floristique et ethnobotanique des plantes sauvages comestibles dans le Département de Bondoukou (Nord-Est de la Côte d'Ivoire). Journal of Applied Biosciences, 98, pp. 9284-9300. https://doi : 10.4314/jab.v98i1.5

47. Ozer, P., Hountondji, Y., Niang, A.J., Karimoune, S., Manzo, O.L. \& Salmon, M. (2010) - Désertification au Sahel : historique et perspectives. BSGLg, 54, pp. 69-84 http://hdl.handle.net/2268/10347 
48. Samuels, J. (2015) - Biodiversity of food species of the Solanaceae family: a preliminary taxonomic inventory of subfamily Solanoideae. Resources, 4, 277-322. https://doi.org/10.3390/resources4020277

49. Sinha, N.K. (2011) - Handbook of Vegetables and Vegetable Processing. Blackwell publishing Ltd: England; 788 p.

50. Ta Bi, I.H., N'Guessan, K., Bomisso, E.L., Assa, R.R. \& Aké, S. (2016) - Etude ethnobotanique de quelques espèces du genre Corchorus Rencontrées En Côte d'Ivoire. European Scientific Journal. 12, pp. 415-431.

51. Touré, O. \& Seck, S.M. (2005) - Exploitations familiales et entreprises agricoles dans la zone des Niayes au Sénégal. International Institute for Environment and Development. Programme Zonzq Arides. Dossier $\mathrm{N}^{\circ} 133.66 \mathrm{p}$

52. Vollbrecht, E. \& Sigmon, B. (2005) - Amazing grass: developmental genetics of maize domestication. Bioch. Soc. Trans. 33: 1502-1506. 